\title{
Production Monitoring to Improve Test Suites
}

\author{
Deepika Tiwari, Long Zhang, Martin Monperrus, and Benoit Baudry \\ KTH Royal Institute of Technology, Sweden
}

\begin{abstract}
In this paper, we propose to use production executions to improve the quality of testing for certain methods of interest for developers. These methods can be methods that are not covered by the existing test suite, or methods that are poorly tested. We devise an approach called PANKTI which monitors applications as they execute in production, and then automatically generates differential unit tests, as well as derived oracles, from the collected data. PANKTI's monitoring and generation focuses on one single programming language, Java. We evaluate it on three real-world, open-source projects: a videoconferencing system, a PDF manipulation library, and an e-commerce application. We show that PANKTI is able to generate differential unit tests by monitoring target methods in production, and that the generated tests improve the quality of the test suite of the application under consideration.
\end{abstract}

Index Terms-Production monitoring, test improvement, test quality, test generation, test oracle

\section{INTRODUCTION}

$\mathbf{S}$ OFTWARE developers write unit tests to assess programs with respect to an expected behavior captured in the form of a test oracle. Yet, the development of strong test suites is challenging: the selection of test data from complex input spaces is hard [1], the specification of good test oracles is costly [2], [3], and the assessment of test quality is timeconsuming [4], [5]. Test improvement has recently emerged as one way to assist developers in these tasks [6]. The key idea is to combine developer-written tests with automatic analysis and generation techniques to complement these developerwritten tests. For example, one can consolidate test oracles with additional method calls [7], or one can augment test inputs through operational abstraction [8]. Our intuition is that we can also use production data to cope with the weaknesses of developer-written tests.

In this paper, we introduce a novel approach called PANKTI for improving test suites with observations collected in production. PANKTI monitors an application in production, to collect execution data before and after certain methods of interest are executed, called target methods in this paper. PANKTI subsequently extracts test input data and derived oracles [2] from the collected data, and finally generates new test cases. The motivation of using production data is that production workloads may exercise behaviors not observed during the test execution [9]. PANKTI targets new test cases towards specific methods that are selected by the developers, according to what they need. For example, developers can decide to target methods that are weakly-tested, as determined by code coverage [10], [11] or mutation testing [12], [13].

PANKTI works as follows, it first instruments the application to monitor the execution of target methods. The application is then deployed in production and executed according to some workload. For each invocation of a target method, the receiving object, the objects passed as arguments, as well as the returned object are serialized and stored. Once production monitoring data has been collected and persisted, PANKTI deserializes the collected production objects to extract test inputs and synthesize derived oracles [2] that capture the behavior observed in production. The last step consists in assembling the input and the oracle in a new differential unit test [14] for the target method, that is, a test that is able to capture behavioral change with respect to a reference version. The essence of PANKTI is to recreate, in the generated differential unit test, the behavior observed in production. The final step in the PANKTI test generation process consists in running the new tests to determine if indeed the overall test quality improves.

We implement PANKTI for Java and evaluate it on three realworld, open-source, complex software systems: a videoconferencing system called Jitsi, a PDF manipulation library called PDFBox, and an e-commerce application called Broadleaf. These projects have between $28 \mathrm{~K}$ and $729 \mathrm{~K}$ lines of code and at least one thousand stars on GitHub. For evaluation purposes, we select as targets the methods that are found weakly-tested with respect to extreme mutation analysis [15]. For these experiments, PANKTI targets a total of 86 weaklytested methods, and observes 122,194 invocations of these methods in production. With the objects collected from these invocations, PANKTI generates 14, 222 differential unit tests, of which $13,878(97.6 \%)$ tests pass. Thanks to these PANKTIgenerated tests, the test quality of 53 of $86(61.6 \%)$ target methods is found to have improved per the considered test adequacy criterion. These results show that PANKTI is able to automatically transform data observed in production into differential unit tests that improve the quality of a test suite. To sum up, our contributions are:

- PANKTI, a tool that monitors programs in production and uses the observed data to automatically generate differential unit tests that target weakly-tested methods;

- An evaluation of the tool on three notable, real-world, open-source Java projects run in production: a videoconferencing system, a PDF manipulation library, and an ecommerce system;

- A publicly available open-source implementation for Java $^{1}$, and open science experimental data ${ }^{2}$ for reproducibility and extension by further research.

\footnotetext{
${ }^{1}$ https://github.com/castor-software/pankti

${ }^{2}$ https://doi.org/10.5281/zenodo.4298604
} 
In section II, we describe our approach for test generation by monitoring programs in production. section III describes our evaluation methodology, section IV describes experimental results, section $\mathrm{V}$ discusses key insights and challenges from these results, section VI discusses related work, and section VII concludes the paper.

\section{The Pankti Approach to Test Generation}

This section describes the details of our technical contribution. Our tool, called PANKTI, exploits observations in production in order to generate new tests that complement the test suite of a Java application.

\section{A. Overview}

The key concepts to describe PANKTI are defined below.

Test improvement: Test improvement is the process of strengthening an existing manually-written test suite, to enhance a specific, measurable test adequacy criterion [16], [17]. This is the main goal of PANKTI.

Target methods: PANKTI focuses on specific methods, for which it will generate new tests. Developers are responsible for selecting these methods based on their test adequacy criterion of choice. For example, PANKTI can target methods in the code that are not fully covered by the test suite. We discuss target method selection in subsection II-B.

Production workload: In our study, a production workload is a sequence of inputs and interactions given to the program in production over a certain period [18]. For instance, a production workload of a PDF reader is to open and scroll over a PDF file.

Differential unit test: PANKTI is designed to generate unit tests that are small, clear, and capture a targeted test intention. The PANKTI unit tests all include an oracle that is based on the behavior observed in production. This production-based oracle can be considered as a derived test oracle per the classification of Barr et al. [2]. These unit tests aim to detect behavioral changes between the version monitored for test generation and upcoming revisions of the program, and are thus called "differential unit tests" by Elbaum et al. [14]. To sum up, PANKTI generates differential unit tests with a derived oracle based on production data.

Production object profile: When PANKTI monitors the execution of an application under consideration with a specific workload, it collects one production object profile each time one of the target methods is invoked. We define an object profile as a quadruplet < method, receiving, parameters, result $>$ as follows: given a target method invocation, we monitor the receiving object that the method is invoked on, with the objects or values passed as parameters to the method, if any, as well as the result object or value returned from the method.

Figure 1 gives an overview of the PANKTI pipeline for test generation. Our approach operates over three inputs: the source code of an application; a set of methods for which developers wish to get new tests; and one production workload. The output of PANKTI is a collection of differential unit tests for the target methods that have been invoked at least once while executing the application.

The workflow of PANKTI is structured in three phases. First, PANKTI instruments each target method with binary instrumentation, to collect runtime data about its execution context. Second, PANKTI executes the application with the input workload and collects runtime object profiles for each invocation of the target methods. Third, PANKTI generates differential unit tests for the target methods. When the target methods are selected based on a test adequacy criterion, PANKTI selects the generated tests that improve the test quality for the method, according to that criterion. The key concepts of PANKTI are illustrated in the next section, and the different steps of our pipeline are presented in detail in the following sections.

\section{B. Selection of Target Methods}

PANKTI performs generation of differential unit tests for a subset of the methods of the application, this subset is chosen by the developer and passed as input configuration to PANKTI. Focusing the generation on specific methods is important to ensure the relevance of the generated tests for the developers.

There are different ways for developers to choose a set of target methods. For example, developers might want to target the methods that implement the latest released feature, or to target the methods added or modified in the most recent commit [19]. Developers may manually specify the targets based on any combination of factors [4]. If there exists a test suite for the application, developers can pick as targets the methods that are not covered by this test suite [9], or choose targets based on test inadequacies [20]. For example, a method may be considered as a target if it is covered by the test suite, but has surviving mutants [12], or if no test fails if its body is removed [21]. The test generation process of PANKTI does not depend on how the target methods are selected.

\section{Working Example}

Here we illustrate the concepts of PANKTI with a concrete example, taken from PDFBox ${ }^{3}$, an open-source application to work with PDF files [22]. Listing 1 shows a method called getName extracted from PDFBox. This method accepts four integer arguments, which are used as identifiers for the font name, platform, encoding, and language, respectively. It returns the name of the font corresponding to the integer identifiers, or null if the identifiers are not found. We say that getName is inadequately tested by the original test suite of PDFBox, since replacing its body with the statements return null; , return "A";, or return " "; is undetected by the 7 existing test cases that trigger its execution [21]. This means that getName is weakly-tested because, in spite of being covered by multiple tests, its output is not specified by a single oracle in the test suite [23].

When we run PDFBox with a production workload, we observe that getName gets invoked 650 times, and we collect 563 production object profiles. One of these object profiles is

\footnotetext{
${ }^{3}$ https://github.com/apache/pdfbox/tree/2.0.21
} 
Inputs

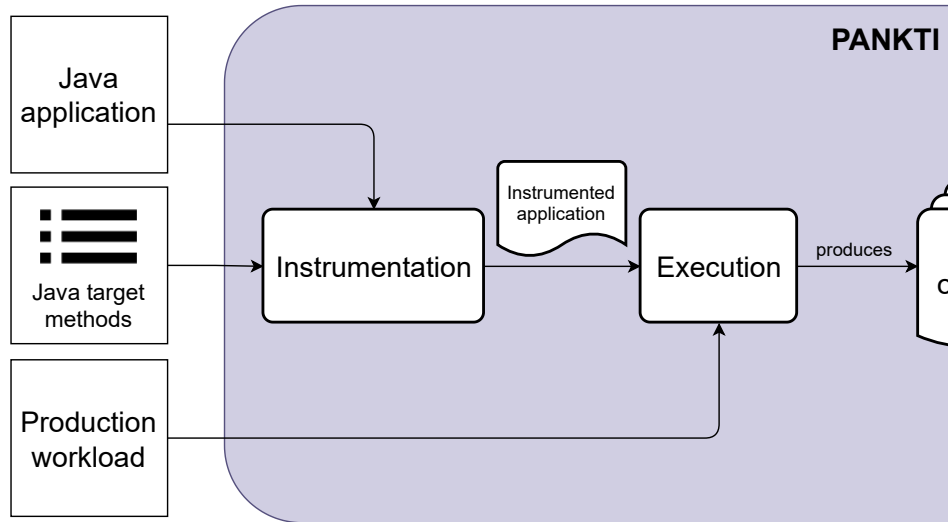

Output
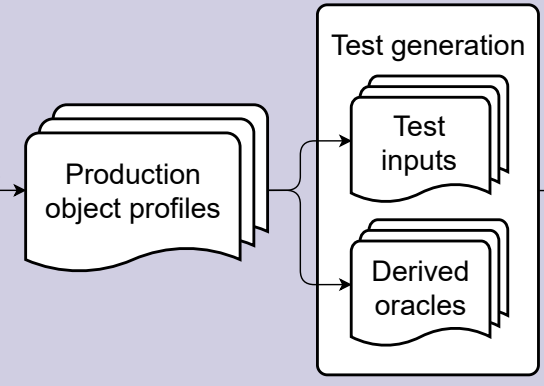

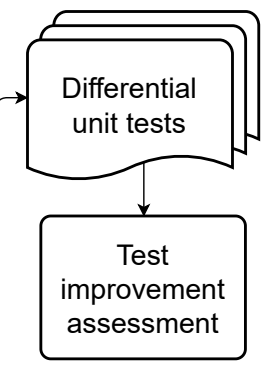

Fig. 1: The PANKTI pipeline: Transforming production workloads into differential unit tests to improve testing of target methods according to a test adequacy criterion.

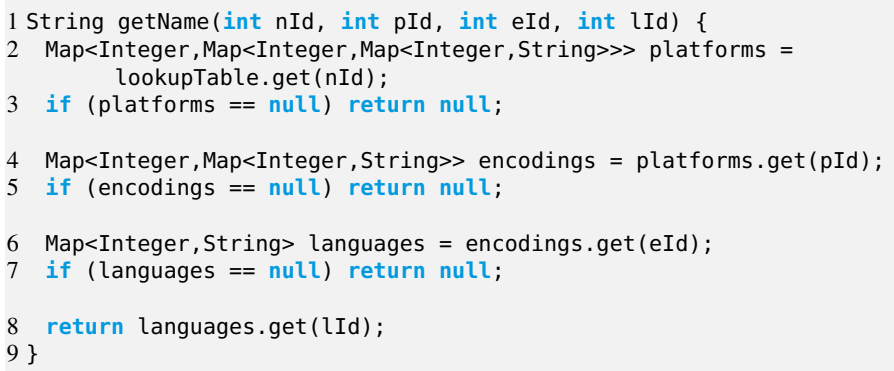

Listing 1: A target method for PANKTI

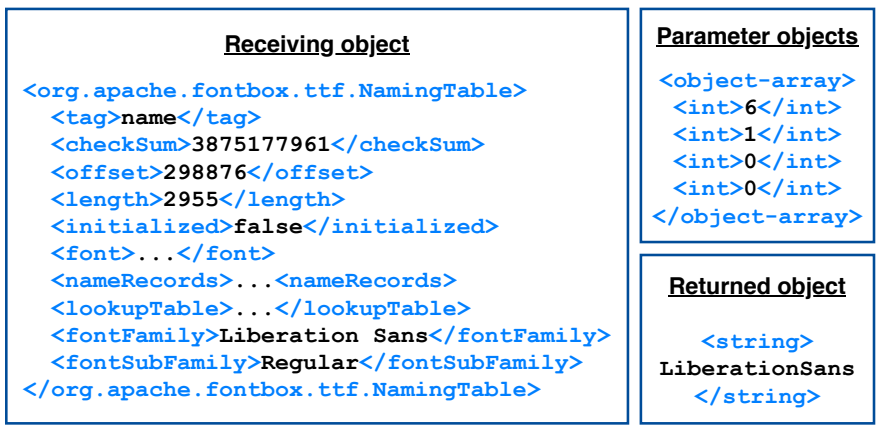

Fig. 2: A production object profile for the target method in Listing 1

illustrated in Figure 2. It includes the receiving object, which is the NamingTable object that getName is invoked on, the four integers passed as parameters to it, and the String object returned as a result from this invocation of getName. All the elements of the object profile are serialized in XML format.

Listing 3 shows a differential unit test generated for getName, based on the production object profile illustrated in Figure 2. In the generated test, each element in the object profile is deserialized into its corresponding object. The generated assertion checks the equality between the String object as observed in production (expected value for this differential unit test), and the result returned from getName invoked on the deserialized NamingTable object, passing the same integer parameters.

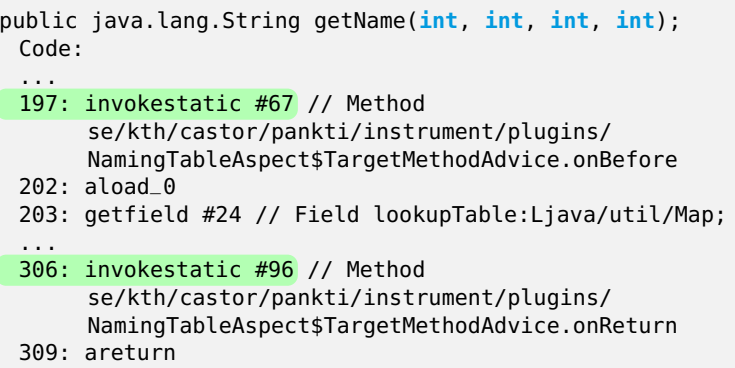

Listing 2: Excerpt showing bytecode transformation of the target method in Listing 1

\section{Execution with Observability}

A unique feature of PANKTI consists in observing the execution of a program in production to collect data for test generation. This execution with observability is articulated around the following three steps.

1) Code Instrumentation: The target methods under consideration are instrumented. The instrumentation of target methods consists of adding all the bytecode instructions required to monitor the execution context, before and just after the method invocation. For instance, the bytecode of the method getName in Listing 1 is transformed into Listing 2, post-instrumentation. The highlighted lines in the excerpt in Listing 2 show the instructions added to the original bytecode of getName. The probe before the method invocation collects a snapshot of the state of the receiving object, i.e., the object the method is being invoked on, as well as the state of any object passed as parameter to the method. The probe after the invocation, saves the object returned by the method.

PANKTI depends on the instrumentation of target methods at the bytecode level in a Java application in production. The key challenge here is to find a robust and reliable framework that can perform this instrumentation at scale, in the production environment.

2) Collection of Production Object Profiles: When the application executes under a specific workload, the instrumentation code for each target method, such as the one in Listing 2, generates one production object profile < 
method, receiving, parameters, result $>$, each time the target method is invoked. PANKTI collects production object profiles in real time, and saves them to the disk. Production objects can be large and complex, with several levels of nesting with sub-objects. Serialization of very deeply nested objects is known to be hard [24], [25]. For this reason, PANKTI requires state-of-the-art serialization technology, which will be presented in II-G. Another crucial requirement of PANKTI is to save the objects in a human-readable format, because they are meant to be used in generated tests read by developers.

3) Selection of Object Profiles: During a pilot experiment, we made two important observations: some target methods are invoked thousands of times as the application executes; some of the serialized objects are very large. For instance, the method toUnicode(int) in PDFBox was invoked 30,840 times with our selected production workload, and the PDTrueTypeFont object it was invoked on had nesting up to 13 levels deep. In production, there is a limited amount of space available to save object profiles. Based on these observations, PANKTI uses a threshold on the maximum size of serialized object profiles. The size threshold is one configuration parameter of PANKTI, for which the default value is $200 \mathrm{MB}$. This implies that object profiles are collected for each invocation of a target method, provided this threshold is not encountered for that method.

\section{E. Generation of Test Cases}

The test generation phase of PANKTI processes the object profiles collected in production to synthesize differential unit tests for the target methods that have been invoked at least once.

We have observed that a target method is often invoked in the same context and the set of collected production object profiles for one specific target method contains redundant data. For instance, the method getName in Listing 1 is invoked 650 times during execution with our selected workload. We collect production object profiles for 563 invocations, as per the size threshold discussed in the previous section. Of these 563 object profiles, 273 are unique. The first step for test generation is to select unique profiles.

Second, for each unique profile, PANKTI generates a differential unit test that follows a systematic template. First, the test case deserializes each part of the object profile: the receiver, the parameters, and the result object. Second, the test case invokes the method on an object initialized with the deserialized receiving and passes the set of deserialized parameters to the method call. Third, we generate an assertion that expects the method invocation to return the same result as observed in production. The serialized representations of objects can vary in size, depending on the complexity of the corresponding object. For the sake of readability, PANKTI includes smaller representations directly in the generated test, but generates resource files for serialized representations that are more sizable. The corresponding objects are then deserialized from these files during the execution of the generated test. This is also the methodology adopted by [26]. For example, PANKTI generated the test case shown in Listing 3, for the method
1 aTest

2 public void testGetName() \{

// generated test for target NamingTable.getName

File fileReceiving = new File("receiving. $x \mathrm{Cl}^{\text {") }}$;

NamingTable receiving0bject $=$ deserialize0bject (fileReceiving)

String returned0bject $=$ "<string $>$ LiberationSans $</$ string $>$ ";

String expected0bject $=$ deserialize0bject ( returned0bject)

assertEquals (expected0bject,

receiving0bject.getName $(6,1,0,0))$;

$10\}$

Listing 3: A generated differential unit test for the target method in Listing 1

in Listing 1, based on the production object profile illustrated in Figure 2. Lines 4 and 5 in the test fetch the receiving object that was observed in production from a resource file and deserialize it. Lines 6 and 7 deserialize the result. Line 8 is the test oracle that asserts an equality between the result that was observed in production and the value returned by the target method getName when it is invoked with the parameters as obtained from the production object profile.

Deserialization of arbitrary objects is a hard problem [24], [25], and can sometimes result in an object that is not identical to the one observed in production. Since PANKTI fully relies on a full serialization-deserialization cycle, we need production grade serialization technology.

\section{F. Test Improvement Assessment}

The final step of the PANKTI pipeline assesses and filters the test cases generated for each target method. Here, we check three aspects: 1) that the generated test passes (see discussion in subsection V-A), 2) that it is not flaky ${ }^{4}$, and 3) that the test improves the test adequacy criterion originally used to select the target methods.

The latter action is performed only if the application has a test suite and if the target methods were selected based on a test inadequacy criterion. In this case, the quality of the test suite before and after the test generation process is compared, per the test inadequacy criterion. If the quality is found to have improved, PANKTI is considered successful in generating a useful test.

\section{G. Implementation}

PANKTI is implemented in Java. By default, PANKTI focuses on generating tests for public and non-static methods. The focus on public methods provides a clear intention for the generated tests, similar to what developers do in the original test suite. Since PANKTI collects object profiles to generate the test inputs and the oracle, we do not target static methods in order to focus the test generation on the invocation of methods on objects and not classes.

PANKTI uses Glowroot ${ }^{5}$ for code instrumentation and observability. Glowroot is an open-source, robust, and lightweight application performance management tool. PANKTI extends its production monitoring and instrumentation capabilities using its Plugin API.

\footnotetext{
${ }^{4}$ we run it 5 times per [27]

${ }^{5}$ https://glowroot.org/
} 
The serialization and deserialization of production object profiles rely on the XStream library ${ }^{6}$. XStream is a state-ofthe art library for serialization, is very robust, can handle arbitrarily complex objects, and allows the registration of custom converters. This library has also been proven to be an effective serialization tool in previous research [14], [28], [29]. Through a parameter, PANKTI can be configured to generate tests with serialized object strings in XML, JSON, and all other formats supported by XStream.

The test synthesis phase relies on Spoon [30] for test code generation. Spoon is a code analysis and transformation library for Java programs. PANKTI leverages the code generation features of Spoon to generate tests that are syntactically correct.

In the last phase, we assess the quality of the tests by compiling and running them. If the target methods are selected based on a test adequacy criterion, we determine if the PANKTI tests improve the test suite with respect to the same criterion.

PANKTI is made publicly available for facilitating reproduction and future research on the topic, at https://github.com/ castor-software/pankti/.

\section{Evaluation Methodology}

This section describes our approach to evaluate PANKTI on real-world software systems. We discuss the criteria used to select the study subjects, their target methods and workloads, as well as the metrics computed for these projects.

\section{A. Study Subjects}

We perform a case-based research study [31] to evaluate PANKTI. We select a set of study subjects according to the following criteria: 1) The project is a real-world, open-source product implemented in Java, built with Maven, and has a test suite; 2) The project has more than 1000 stars and more than 1000 commits, which is indicative of a popular and mature project; 3) The project builds, the test suite passes, and the project can be deployed with the computing resources of our research lab; 4) A realistic production workload for the project can be executed in our laboratory environment.

We manually search for projects on GitHub that meet all of the above criteria, and select 3 of them. Jitsi/Jicofo is a component used in the Jitsi Meet videoconference system, a highquality conferencing solution which supports audio, video, and text communication. Jicofo is responsible for maintaining the connection of the participants of a Jitsi Meet conference. PDFBox is a robust and mature project by the Apache Software Foundation for producing and reading PDF documents such as legal decisions, invoices, and contracts. This PDF application provides capabilities such as digital signatures, image conversion, etc. It includes standalone command-line tools that can be invoked on PDF and text documents [22]. Our third subject is BroadleafCommerce (henceforth referred to as Broadleaf), which is an enterprise e-commerce framework based on Spring. Broadleaf provides all necessary components for a web shopping experience, such as user and product management, a front-end website, and API services.

\footnotetext{
${ }^{6}$ http://x-stream.github.io/
}

TABLE I: Real-world projects used in our experiments

\begin{tabular}{llrcr}
\hline PROJECT & VERSION & \#STARS & \#COMMITS & LOC \\
\hline Jitsi/Jicofo & ${\text { stable- } 4857^{7}}^{7}$ & $14 \mathrm{~K}$ & 1,248 & $28.32 \mathrm{~K}$ \\
PDFBox & $2.0 .21^{8}$ & $1.3 \mathrm{~K}$ & 8,244 & $728.8 \mathrm{~K}$ \\
Broadleaf & $6.1 .4-\mathrm{GA}^{9}$ & $1.4 \mathrm{~K}$ & 16,964 & $197.5 \mathrm{~K}$ \\
\hline
\end{tabular}

The version, number of stars (\#STARS), number of commits (\#COMMITS), and lines of code (LOC) for each of these projects are shown in Table I. The three projects have between $28 \mathrm{~K}$ and $729 \mathrm{~K}$ lines of code.

\section{B. Experiments with Production Workloads}

To generate differential unit tests using production data for each of our study subjects, we execute them in production conditions that are realistic, relevant, and reliable. This section describes our choice of workloads for each of the study subjects.

1) Production workloads for Jitsi/Jicofo: We hold a videoconference meeting to obtain a Jitsi/Jicofo workload. For this, we deploy the full Jitsi stack using the docker-jitsi-meet project. We create a meeting room for three members of the research lab for the experiment. The members join this meeting room from different IP addresses to have an hourlong meeting. During the meeting, all the participants turn on their camera and microphone. Each participant also sends at least one text message via the chat box.

2) Production workloads for PDFBox: As documentation and archival become more digitized across all domains, the Portable Document Format, or PDF, has emerged as a standard to ensure platform-independence and interoperability with multiple user environments. To select realistic and non-trivial workloads to experiment with PDFBox, we shortlist five PDF documents from GovDocs1 [32], an online corpora of PDF files made available for research and analysis. The same protocol has also been followed in previous research [33]. In order to have a good trade-off between the diversity of the files and the execution time for the experiments, we select the PDF documents based on the following criteria: 1) They have between 1 to 5 pages of text; 2) They include at least one image or photograph; and 3) Their size does not exceed 1.5 MB. The five documents that we use as part of the PDFBox workload are available as part of our replication package.

For each of the five selected documents, we invoke ten PDFBox features, which include the encryption of the PDF document with a password, decryption of an encrypted PDF document, extraction of text and images from a PDF document, etc. In summary, the workload to evaluate PANKTI on PDFBox is composed of $5 \times 10$ different invocations to the PDFBox command line tool.

3) Production workloads for Broadleaf: The workloads for Broadleaf consist of general steps to purchase products online as an end-user: a user visits the homepage of the website

\footnotetext{
${ }^{7}$ https://github.com/jitsi/jicofo/tree/stable/jitsi-meet_4857

${ }^{8}$ https://github.com/apache/pdfbox/tree/2.0.21

${ }^{9}$ https://github.com/BroadleafCommerce/BroadleafCommerce/tree/ broadleaf-6.1.4-GA
} 
first, then registers a new account and logs in, then views the web page to add some products into the shopping cart, and finally the user checks out the products and logs out. There are 871 HTTP requests in the workload, comprising of 865 GET requests and 6 POST requests. The GET requests are used to fetch product information and necessary files, such as JavaScript and CSS files. The POST requests are mainly for user registration, logging in, checking out products, and logging out.

\section{Experiments with System Tests}

System tests are typically designed to verify end-to-end uses of applications. They may exercise multiple and complex scenarios [34] or use cases [35] through the system. It is possible to generate differential unit tests from system tests, as proposed by the seminal papers of Elbaum et al. [14] and Saff et al. [36]. Inspired by this related work in the area of test improvement, we perform an experiment where we use PANKTI with system tests, instead of a production workload.

The goal of this experiment is to demonstrate the ability of PANKTI to generate unit tests by observing the execution of systems tests. So first, we need to select system tests. However, there is no strict definition of what constitutes a system test. Per previous research, we note that system tests and unit tests differ in the extent of the application they exercise, or the number of lines of code they cover [37]. Consequently, as actionable definition, we define system tests as those tests in the original test suite of our study subjects that cover at least 1500 lines of code in the application (this threshold of 1500 lines of code to identify system tests was used in previous research [38]). We measure the line coverage of each test with $\mathrm{JaCoCo}^{10}$, in order to select the ones we consider as system tests.

We pass those system tests to PANKTI, which collects object profiles whenever target methods are invoked during their execution. The collected object profiles from system tests are then used to generate differential unit tests. The results of using PANKTI to carve differential unit tests from system tests will be discussed in subsection IV-E

\section{Target Methods for The Evaluation}

For our evaluation, we use "pseudo-tested methods" as the test adequacy criterion [15], [21] to select target methods in the three projects. A method is said to be pseudo-tested if it is invoked at least once when running the test suite, but no test fails if its body is removed. This means that no existing test is able to detect extreme transformations of these methods. Pseudo-testedness is a state-of-the-art criterion that developers care about, as evaluated in user studies [21]. We use the Descartes ${ }^{11}$ tool to automatically identify pseudo-tested methods in the three study subjects.

This choice of targets for the evaluation implies a very clear way to assess the improvement provided by the PANKTI differential unit tests. A test for a specific target method is

\footnotetext{
${ }^{10} \mathrm{https}: / /$ www.eclemma.org/jacoco/

${ }^{11}$ https://github.com/STAMP-project/pitest-descartes
}

considered to be an improvement if the target method is no longer pseudo-tested.

\section{E. Measurements}

In this section, we introduce the metrics that we collect as part of our case-based study and assessment of PANKTI.

Testing metrics. First, we compute the following quantitative metrics capturing the test quality of the subjects:

1) Test suite method coverage, TMCov, represents the percentage of methods in the project that are covered by at least one test case in the original test suite.

2) Test suite line coverage, TLCov, represents the percentage of the lines of code in the project covered by at least one test case in the original test suite.

3) Workload method coverage, WMCov, determines the percentage of methods in the project that are invoked when we execute the project with our selected production workload. 4) Workload line coverage, WLCov, determines the percentage of lines of code in the project that are executed with the selected workload.

5) Target methods, \#TARGET, is the number of target methods in the project. In the case of our evaluation, this is the number of pseudo-tested methods, i.e., the methods that are covered by at least one test case in the original test suite, but no test case fails if their body is replaced with a statement returning a default value; and

6) Effective target methods, \#EFF_TARGET, is the number of target methods for which we use PANKTI to generate units tests. In the case of the experiments with production workloads, this is the number of pseudo-tested methods that are invoked when running the workload. In the case of the experiments with system tests, this is the number of pseudotested methods that are invoked when running the system tests.

Metrics TMCov and TLCov are indicators of the quality of the test suite of the project. Metrics WMCov and WLCov indicate the proportion of the project that is covered by our selected production workload. Metrics \#TARGET and \#EFF_TARGET are computed specifically as starting points for PANKTI. We use JaCoCo to obtain the coverage. \#TARGET is computed with Descartes [21], while \#EFF_TARGET is the subset of \#TARGET covered during the experiments with production workloads and with system tests.

The second set of metrics we collect aim at assessing the ability of PANKTI at transforming production data into differential unit tests.

7) Target method invocations, \#INVOCATIONS, is the number of invocations of an effective target method as the application executes with the production workload.

8) Collected object profiles, \#COLLECTED, is the number of production object profiles for each effective target method that are captured as the application executes with the selected workload.

9) Unique object profiles, \#UNIQUE, is the size of the subset of collected object profiles that includes unique combinations of the constituent elements.

10) Object profile size, SIZE is the size on disk of collected production object profiles. 
11) Differential unit tests, \#PANKTI_TESTS, is the number of test cases generated by PANKTI using the \#UNIQUE object profiles.

12) Passing test cases, \#PASSING, is the number of generated tests that pass when executed.

13) Failing test cases, \#FAILING, is the number of generated tests that do not pass when executed.

14) PANKTI_STATUS (pseudo-tested or well-tested) is the classification of an effective target method, as obtained by Descartes, after the addition of the generated test to the test suite of the project. After adding the PANKTI-generated tests in the application test suite, a target method is either still pseudotested, or, is not pseudo-tested anymore. In the latter case, we give it the status of well-tested.

Metrics \#INVOCATIONS, \#COLLECTED, \#UNIQUE, and SIZE are intermediate outputs of PANKTI as it monitors the target methods in the project in the production environment. Metrics \#PANKTI_TESTS, \#PASSING, \#FAILING, and PANKTI_STATUS are the final outputs of PANKTI for the project, and are indicators of its success in generating differential unit tests for the target methods in the project.

\section{EXPERIMENTAL RESULTS}

This section discusses our findings when running PANKTI on our three study subjects. First, we present general data about the quality of the subjects' test suites. The subsequent sections discuss each case study separately.

\section{A. Descriptive Statistics}

Table II summarizes the key metrics about the original test suite and about the workload: TMCov, TLCov, WMCov, WLCov, \#TARGET, and \#EFF_TARGET (see subsection III-E). Let us take the case of Jitsi/Jicofo as an example. The original test suite of Jitsi/Jicofo covers $49.4 \%$ of the methods, and $46.7 \%$ of the lines in the project. Our selected production workload for Jitsi/Jicofo covers $48.9 \%$ of the methods and $46.2 \%$ of the lines in the project. We find that 29 public and non-static methods are pseudo-tested in Jitsi/Jicofo (\#TARGET). This means that they are covered by at least one test in the suite, but no test is able to detect extreme mutations in these methods. When we instrument these 29 methods and observe Jitsi in production, we find that all 29 of them get invoked (\#EFF_TARGET).

Per Table II, the original test suite of all the three projects varies in coverage, with a highest line coverage of $53.5 \%$. Moreover, the coverage achieved by the workload is lower than the test coverage for all of the three cases. This observation is consistent with the findings of Wang et al. [9].

Pseudo-tested methods are present in all projects, confirming the results of Vera-Perez et al. [21] about the prevalence of such methods. These methods, covered by the original test suite, but weakly-tested, provide a false sense of trust in the test suite, which we aim at mitigating with new test cases generated by PANKTI.

On executing each project with its corresponding workload, some of these pseudo-tested methods are indeed invoked in production. This is indicated by the column "\#EFF_TARGET"

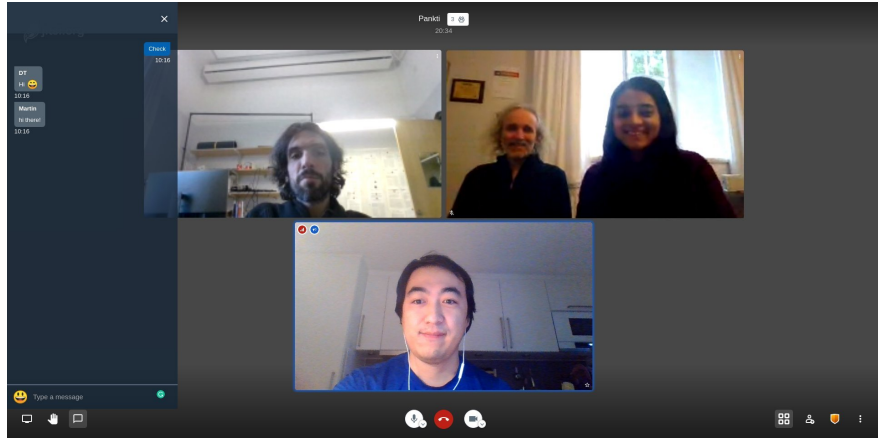

Fig. 3: A Jitsi Meeting with PANKTI Attached

in Table II. This is the baseline that PANKTI aims to improve: we want to generate tests such that some of those pseudotested methods become well-tested: 29, 46, and 11 target methods for Jitsi/Jicofo, PDFBox, and Broadleaf, respectively.

\section{B. Case Study 1: Jitsi/Jicofo}

PANKTI targets 29 pseudo-tested methods in Jitsi/Jicofo for instrumentation. Then, we run Jitsi for a videoconference during which we collect object profiles. We, the authors, perform this videoconference to discuss this paper. Figure 3 presents a screenshot of this meeting, where PANKTI was attached to the Jitsi/Jicofo component. During the one-hour meeting, target methods keep getting invoked periodically, triggering object profile collection, yet no participant observes a degradation of Jitsi's user experience such as a delay, or a crash.

We now discuss the results of PANKTI on Jitsi, presented in Table III. Table III is grouped by the study subject and sorted by the invocation count of the target methods. Each row corresponds to one effective target method, and includes the values of the metrics introduced in subsection III-E. For each of these methods, Table III shows the number of times it was invoked with the production workload (\#INVOCATIONS), the number of object profiles corresponding to these invocations that are serialized (\#COLLECTED), the number of collected object profiles that are unique (\#UNIQUE), as well as the number of differential unit tests generated by PANKTI (\#PANKTI_TESTS). Of the tests generated, the number of tests that pass (\#PASSING), and the ones that do not (\#FAILING), are also indicated. The Descartes classification (PANKTI_STATUS) of the target method after the addition of the generated tests to the test suite of the project is shown in the last column, it either remains the same as before, i.e., "pseudo-tested", or upgrades to "well-tested".

Rows 1 to 29 of Table III show the experimental results for Jitsi. The 29 effective target methods are invoked 152 times during the experiment. PANKTI collects 110 object profiles, 20 of which are unique. Based on the unique object profiles, PANKTI generates 20 differential unit tests in total for Jitsi/Jicofo. All of the generated tests pass and improve the quality of the Jitsi/Jicofo test suite, transforming 19/29 $(65.5 \%)$ pseudo-tested methods into well-tested ones.

Let us look at one successful case. Listing 4 is the source code of method 
TABLE II: Original test suite method and line coverage (TMCov, TLCov), workload method and line coverage (WMCov, WLCov), number of pseudo-tested methods (\#TARGET) and number of pseudo-tested methods invoked when running the workload (\#EFF_TARGET) for each study subject

\begin{tabular}{lrrrrrr}
\hline PROJECT & TMCov & TLCov & WMCov & WLCov & \#TARGET & \#EFF_TARGET \\
\hline \multirow{2}{*}{ Jitsi/Jicofo } & $49.4 \%$ & $46.7 \%$ & $48.9 \%$ & $46.2 \%$ & \multirow{2}{*}{29} & \\
\hline \multirow{2}{*}{ PDFBox } & $(667 / 1,350)$ & $(3,537 / 7,571)$ & $(660 / 1,350)$ & $(3,500 / 7,571)$ & 29 \\
\hline \multirow{2}{*}{ Broadleaf } & $54.8 \%$ & $53.5 \%$ & $21.6 \%$ & $21.0 \%$ & \multirow{2}{*}{138} & 46 \\
& $(6,049 / 11,042)$ & $(34,653 / 64,787)$ & $(2,390 / 11,042)$ & $(13,630 / 64,787)$ & $19.2 \%$ & \multirow{2}{*}{11} \\
\hline
\end{tabular}

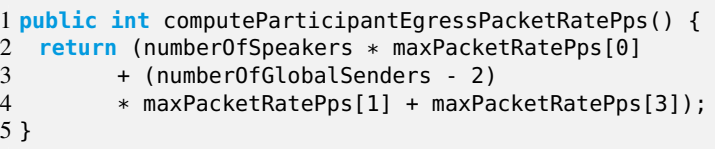
Jitsi/Jicofo

\begin{tabular}{|c|c|}
\hline 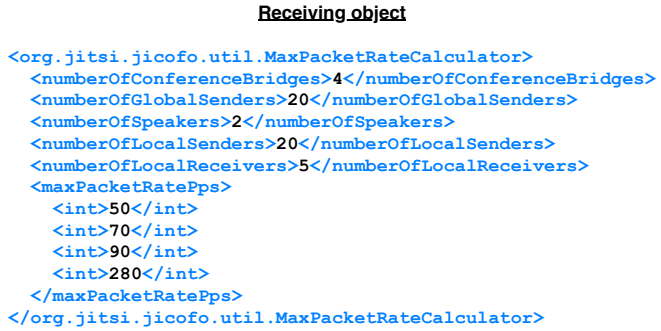 & $\begin{array}{l}\text { Parameter objects } \\
\text { <object-array/> }\end{array}$ \\
\hline
\end{tabular}

Fig. 4: A production object profile for the method computeParticipantEgressPacketRatePps() in Jitsi/Jicofo

computeParticipantEgressPacketRatePps

(row

27 in Table III) in class MaxPacketRateCalculator. The method is covered by developer-written unit tests, but is identified as pseudo-tested: if the method body is replaced with return $-1 ;$, return $0 ;$, or return 1 ; , no test in the original test suite fails. By observing the behavior of this method in production, PANKTI collects the object profile shown in Figure 4. A new differential unit test is then generated based on this profile, shown in Listing 5. The generated test includes an explicit assertion for the behavior of computeParticipantEgressPacketRatePps. After adding this generated differential unit test to the test suite, the method is not pseudo-tested anymore.

During the object profile collection phase, PANKTI collects 110 object profiles for 20 out of $29(70.0 \%)$ methods. This corresponds to a total of $9.79 \mathrm{~KB}$ collected data, for object profiles that vary between $0.48 \mathrm{~KB}$ and $0.50 \mathrm{~KB}$, with a median value of $0.49 \mathrm{~KB}$. For the duration of our experiment, none of the methods reach the threshold of the file size (200 $\mathrm{MB})$ for their object profile collection.

We observe that PANKTI collects no object profile for 9 methods, and does not collect all the profiles for 3 methods (rows 1, 3, and 4). This occurs when PANKTI faces extreme situations for object serialization at runtime: 1) A ConcurrentModificationException happens if
1 @Test

2 public void testCPEgressPacketRatePps() \{

3 // content of the serialized receiving object from Fig. 4

String receivingobjectStr =

"<org.jitsi.jicofo.util. MaxPacketRateCalculator $>$. .

</org. jitsi. jicofo, util . MaxPacketRateCalculator>" ,

5 MaxPacketRateCalculator receiving0bject $=$ deserialize0bject (receiving0bjectStr)

6 assertEquals $(1640$, $7\}$ receiving0bject. computeParticipantEgressPacketRatePps());

Listing 5: A generated differential unit test for the computeParticipantEgressPacketRatePps method

XStream tries to serialize an object that is being changed. This may happen if the object contains some fields which are accessed by multiple threads frequently. 2) A "no converter specified" exception happens if XStream does not know how to serialize some members in the object. This case only happens when XStream fails to serialize class NetworkAddressManagerServiceImpl in the package net. java.sip. communicator. impl. netaddr of Jitsi/Jicofo. This is due to the fact that this class contains fields that are related to system resources such as sockets and threads, which are fundamentally unserializable. 3) a "security rule violation" happens if XStream refuses to serialize a specific class which is protected by security code. Overall, this clearly show that serialization of arbitrary objects is fundamentally hard, and not yet solved by the stateof-the-art of serialization. After the profile collection phase, PANKTI successfully generates test cases for all of the covered methods. All these new test cases pass.

\section{Highlight from the Jitsi/Jicofo Experiment}

The experiment on Jitsi/Jicofo uses a production workload for videoconferencing, an essential feature of remote working and social interactions in 2020 and 2021. PANKTI successfully collects 110 object profiles for 20 methods. PANKTI generates 20 new test cases that improve the testing of 19 out of 29 target methods of Jitsi. This experiment shows that PANKTI does not perturb user experience, users can have a smooth videoconference while generating tests.

\section{Case Study 2: PDFBox}

There are 138 pseudo-tested methods in PDFBox. The production workload covers 46 of these methods, which all 
TABLE III: Experimental results from executing production workloads of the study subjects used to evaluate PANKTI

\begin{tabular}{|c|c|c|c|c|c|c|c|}
\hline \# & CLASS_METHOD & \#INVOCATIONS & \#COLLECTED & \#UNIQUE & \#PANKTI_TESTS & \#PASSING & \#FAILING \\
\hline 1 & JitsiMeetConfig_isTccEnabled & 20 & $18 / 20(90 \%)$ & $1 / 18(0.05 \%)$ & 1 & $1 / 1(100 \%)$ & $0 / 1(0 \%)$ \\
\hline 2 & JitsiMeetConfig_isOctoEnabled & 15 & $15 / 15(100 \%)$ & $1 / 15(0.06 \%)$ & 1 & $1 / 1(100 \%)$ & $0 / 1(0 \%)$ \\
\hline 3 & JitsiMeetConfig_getMinBitrate & 12 & $11 / 12(91.6 \%)$ & $1 / 11(0.09 \%)$ & 1 & $1 / 1(100 \%)$ & $0 / 1(0 \%)$ \\
\hline 4 & JitsiMeetConfig_getStartBitrate & 12 & $11 / 12(91.6 \%)$ & $1 / 11(0.09 \%)$ & 1 & $1 / 1(100 \%)$ & $0 / 1(0 \%)$ \\
\hline 5 & JitsiMeetConfig__isRembEnabled & 12 & $12 / 12(100 \%)$ & $1 / 12(0.08 \%)$ & 1 & $1 / 1(100 \%)$ & $0 / 1(0 \%)$ \\
\hline 6 & JitsiMeetServices_getJigasiDetector & 8 & $0 / 8(0 \%)$ & 0 & 0 & 0 & 0 \\
\hline 7 & JitsiMeetServices_getJibriDetector & 6 & $0 / 6(0 \%)$ & 0 & 0 & 0 & 0 \\
\hline 8 & JitsiMeetConfig_getMinParticipants & 5 & $5 / 5(100 \%)$ & $1 / 5(20 \%)$ & 1 & $1 / 1(100 \%)$ & $0 / 1(0 \%)$ \\
\hline 9 & ColibriConferenceImpl_hasJustAllocated & 4 & $0 / 4(0 \%)$ & 0 & 0 & 0 & 0 \\
\hline 10 & JitsiMeetConfig_getEnforcedVideobridge & 4 & $4 / 4(100 \%)$ & $1 / 4(25 \%)$ & 1 & $1 / 1(100 \%)$ & $0 / 1(0 \%)$ \\
\hline 11 & JitsiMeetConfig_getOpusMaxAverageBitrate & 4 & $4 / 4(100 \%)$ & $1 / 4(25 \%)$ & 1 & $1 / 1(100 \%)$ & $0 / 1(0 \%)$ \\
\hline 12 & JitsiMeetConfig_getStartAudioMuted & 4 & $4 / 4(100 \%)$ & $1 / 4(25 \%)$ & 1 & $1 / 1(100 \%)$ & $0 / 1(0 \%)$ \\
\hline 13 & JitsiMeetConfig_getStartVideoMuted & 4 & $4 / 4(100 \%)$ & $1 / 4(25 \%)$ & 1 & $1 / 1(100 \%)$ & $0 / 1(0 \%)$ \\
\hline 14 & JitsiMeetConfig_isRtxEnabled & 4 & $4 / 4(100 \%)$ & $1 / 4(25 \%)$ & 1 & $1 / 1(100 \%)$ & $0 / 1(0 \%)$ \\
\hline 15 & JitsiMeetConfig_stereoEnabled & 4 & $4 / 4(100 \%)$ & $1 / 4(25 \%)$ & 1 & $1 / 1(100 \%)$ & $0 / 1(0 \%)$ \\
\hline 16 & Participant_hasDtlsSupport & 4 & $0 / 4(0 \%)$ & 0 & 0 & 0 & 0 \\
\hline 17 & Participant_hasIceSupport & 4 & $0 / 4(0 \%)$ & 0 & 0 & 0 & 0 \\
\hline 18 & Participant_hasRtxSupport & 4 & $0 / 4(0 \%)$ & 0 & 0 & 0 & 0 \\
\hline 19 & ProtocolProviderHandler_getXmppConnection & 4 & $0 / 4(0 \%)$ & 0 & 0 & 0 & 0 \\
\hline 20 & JitsiMeetConfig_isLipSyncEnabled & 3 & $3 / 3(100 \%)$ & $1 / 3(33.3 \%)$ & 1 & $1 / 1(100 \%)$ & $0 / 1(0 \%)$ \\
\hline 21 & JitsiMeetConfig_useRoomAsSharedDocName & 3 & $3 / 3(100 \%)$ & $1 / 3(33.3 \%)$ & 1 & $1 / 1(100 \%)$ & $0 / 1(0 \%)$ \\
\hline 22 & JitsiMeetServices_getSipJibriDetector & 3 & $0 / 3(0 \%)$ & 0 & 0 & 0 & 0 \\
\hline 23 & JitsiMeetConfig_getAudioPacketDelay & 2 & $2 / 2(100 \%)$ & $1 / 2(50 \%)$ & 1 & $1 / 1(100 \%)$ & $0 / 1(0 \%)$ \\
\hline 24 & JitsiMeetConfig_getChannelLastN & 2 & $2 / 2(100 \%)$ & $1 / 2(50 \%)$ & 1 & $1 / 1(100 \%)$ & $0 / 1(0 \%)$ \\
\hline 25 & JitsiMeetConferenceImplSBridgeSession_terminate & 1 & $0 / 1(0 \%)$ & 0 & 0 & 0 & 0 \\
\hline 26 & MaxPacketRateCalculator_computeEgressPacketRatePps & 1 & $1 / 1(100 \%)$ & $1 / 1(100 \%)$ & 1 & $1 / 1(100 \%)$ & $0 / 1(0 \%)$ \\
\hline 27 & MaxPacketRateCalculator_computeIngressPacketRatePps & 1 & $1 / 1(100 \%)$ & $1 / 1(100 \%)$ & 1 & $1 / 1(100 \%)$ & $0 / 1(0 \%)$ \\
\hline 28 & MaxPacketRateCalculator_computeParticipantEgressPacketRatePps & 1 & $1 / 1(100 \%)$ & $1 / 1(100 \%)$ & 1 & $1 / 1(100 \%)$ & $0 / 1(0 \%)$ \\
\hline 29 & MaxPacketRateCalculator_computeSenderIngressPacketRatePps & 1 & $1 / 1(100 \%)$ & $1 / 1(100 \%)$ & 1 & $1 / 1(100 \%)$ & $0 / 1(0 \%)$ \\
\hline & JICOFO_TOTAL & 152 & $110 / 152(72.4 \%)$ & $20 / 110(18.2 \%)$ & 20 & $20 / 20(100 \%)$ & $0 / 20(0 \%)$ \\
\hline 30 & RenderingMode_isFill & 30944 & $30944 / 30944(100 \%)$ & $1 / 30944(0.00003 \%)$ & 1 & $1 / 1(100 \%)$ & $0 / 1(0 \%)$ \\
\hline 31 & PDSimpleFont_toUnicode & 30840 & $37 / 30840(0.001 \%)$ & $35 / 37(94.5 \%)$ & 35 & $35 / 35(100 \%)$ & $0 / 35(0 \%)$ \\
\hline 32 & GlyfSimpleDescript_getFlags & 23471 & $23471 / 23471(100 \%)$ & $11730 / 23471(49.9 \%)$ & 11730 & $11730 / 11730(100 \%)$ & $0 / 11730(0 \%)$ \\
\hline 33 & TTFGlyph2D_getPathForCharacterCode & 15472 & $\mathbf{3 4} / 15472(0.002 \%)$ & $34 / 34(100 \%)$ & 34 & $0 / 34(0 \%)$ & $34 / 34(100 \%)$ \\
\hline 34 & TTFGlyph2D_getPathForGID & 15472 & $34 / 15472(0.002 \%)$ & $34 / 34(100 \%)$ & 34 & $0 / 34(0 \%)$ & $34 / 34(100 \%)$ \\
\hline 35 & PDTrueTypeFont_getPath & 782 & $36 / 782(0.04 \%)$ & $36 / 36(100 \%)$ & 36 & $0 / 36(0 \%)$ & $36 / 36(100 \%)$ \\
\hline 36 & NamingTable_getName & 650 & $\mathbf{5 6 3} / 650(86.6 \%)$ & 273 / $563(48.5 \%)$ & 273 & 273 / $273(100 \%)$ & $0 / 273(0 \%)$ \\
\hline 37 & DefaultResourceCache_getFont & 546 & $29 / 546(0.05 \%)$ & $29 / 29(100 \%)$ & 29 & $5 / 29(0.17 \%)$ & $24 / 29(82.7 \%)$ \\
\hline 38 & HorizontalMetricsTable_getLeftSideBearing & 431 & $360 / 431(83.5 \%)$ & $360 / 360(100 \%)$ & 360 & $360 / 360(100 \%)$ & $0 / 360(0 \%)$ \\
\hline 39 & TTFDataStream_readUnsignedByteArray & 431 & $431 / 431(100 \%)$ & $431 / 431(100 \%)$ & 431 & $431 / 431(100 \%)$ & $0 / 431(0 \%)$ \\
\hline 40 & HorizontalMetricsTable_getAdvanceWidth & 275 & $275 / 275(100 \%)$ & $174 / 275(63.3 \%)$ & 174 & $174 / 174(100 \%)$ & $0 / 174(0 \%)$ \\
\hline 41 & TrueTypeFont_getAdvanceWidth & 275 & $275 / 275(100 \%)$ & $174 / 275(63.3 \%)$ & 174 & $174 / 174(100 \%)$ & $0 / 174(0 \%)$ \\
\hline 42 & PDSimpleFont_hasExplicitWidth & 274 & 144 / $274(52.5 \%)$ & $117 / 144(81.2 \%)$ & 117 & $117 / 117(100 \%)$ & $0 / 117(0 \%)$ \\
\hline 43 & PDFontDescriptor_getFlags & 227 & $227 / 227(100 \%)$ & $54 / 227(23.8 \%)$ & 54 & $54 / 54(100 \%)$ & $0 / 54(0 \%)$ \\
\hline 44 & FontCache_getFont & 115 & $115 / 115(100 \%)$ & $106 / 115(92.2 \%)$ & 106 & $83 / 106(78.3 \%)$ & $23 / 106(21.7 \%)$ \\
\hline 45 & DefaultResourceCache_getColorSpace & 72 & $25 / 72(34.7 \%)$ & $23 / 25(92 \%)$ & 23 & $4 / 23(17.4 \%)$ & $19 / 23(82.6 \%)$ \\
\hline 46 & DefaultResourceCache_getXObject & 66 & $15 / 66(22.7 \%)$ & $15 / 15(100 \%)$ & 15 & $13 / 15(86.6 \%)$ & $2 / 15(13.3 \%)$ \\
\hline 47 & PDImageXObject_getInterpolate & 66 & $8 / 66(12.1 \%)$ & $8 / 8(100 \%)$ & 8 & $8 / 8(100 \%)$ & $0 / 8(0 \%)$ \\
\hline 48 & PDImageXObject_getMask & 66 & $13 / 66(19.7 \%)$ & $13 / 13(100 \%)$ & 13 & $13 / 13(100 \%)$ & $0 / 13(0 \%)$ \\
\hline 59 & PDCIDFontType2_codeToGID & 58 & 38 / $58(65.5 \%)$ & $7 / 38(18.4 \%)$ & 7 & $7 / 7(100 \%)$ & $0 / 7(0 \%)$ \\
\hline 50 & DefaultResourceCache_getExtGState & 49 & $24 / 49(49 \%)$ & 22 / $24(91.6 \%)$ & 22 & $4 / 22(18.1 \%)$ & $18 / 22(81.8 \%)$ \\
\hline 51 & PDResources_getExtGState & 49 & $19 / 49(38.8 \%)$ & 18 / $19(94.7 \%)$ & 18 & $0 / 18(0 \%)$ & 18 / $18(100 \%)$ \\
\hline 52 & PDExtendedGraphicsState_getStrokingOverprintControl & 48 & $48 / 48(100 \%)$ & $3 / 48(0.06 \%)$ & 3 & $3 / 3(100 \%)$ & $0 / 3(0 \%)$ \\
\hline 53 & COSParser_getEncryption & 46 & $38 / 46(82.6 \%)$ & $33 / 38(86.8 \%)$ & 33 & $13 / 33(39.4 \%)$ & $20 / 33(60.6 \%)$ \\
\hline 54 & XrefTrailerResolver_getXrefType & 46 & $46 / 46(100 \%)$ & $17 / 46(36.9 \%)$ & 17 & $17 / 17(100 \%)$ & $0 / 17(0 \%)$ \\
\hline 55 & PDImageXObject_getColorKeyMask & 39 & $7 / 39(17.9 \%)$ & $7 / 7(100 \%)$ & 7 & $7 / 7(100 \%)$ & $0 / 7(0 \%)$ \\
\hline 56 & PDImageXObject_getDecode & 39 & $7 / 39(17.9 \%)$ & $7 / 7(100 \%)$ & 7 & $7 / 7(100 \%)$ & $0 / 7(0 \%)$ \\
\hline 57 & PDExtendedGraphicsState_getAutomaticStrokeAdjustment & 33 & $33 / 33(100 \%)$ & $4 / 33(12.1 \%)$ & 4 & $4 / 4(100 \%)$ & $0 / 4(0 \%)$ \\
\hline 58 & PDImageXObject_getOptionalContent & 33 & $7 / 33(21.2 \%)$ & $7 / 7(100 \%)$ & 7 & $7 / 7(100 \%)$ & $0 / 7(0 \%)$ \\
\hline 59 & PDExtendedGraphicsState_getNonStrokingOverprintControl & 24 & $24 / 24(100 \%)$ & $3 / 24(12.5 \%)$ & 3 & $3 / 3(100 \%)$ & $0 / 3(0 \%)$ \\
\hline 60 & PDExtendedGraphicsState_getOverprintMode & 24 & $24 / 24(100 \%)$ & $3 / 24(12.5 \%)$ & 3 & $3 / 3(100 \%)$ & $0 / 3(0 \%)$ \\
\hline 61 & PDFontDescriptor_isSymbolic & 24 & $24 / 24(100 \%)$ & $20 / 24(83.3 \%)$ & 20 & $20 / 20(100 \%)$ & $0 / 20(0 \%)$ \\
\hline 62 & PDExtendedGraphicsState_getSmoothnessTolerance & 21 & $21 / 21(100 \%)$ & $3 / 21(14.3 \%)$ & 3 & $3 / 3(100 \%)$ & $0 / 3(0 \%)$ \\
\hline 63 & PDFontDescriptor_isFixedPitch & 21 & $21 / 21(100 \%)$ & $6 / 21(28.6 \%)$ & 6 & $6 / 6(100 \%)$ & $0 / 6(0 \%)$ \\
\hline 64 & PDFontDescriptor_isttalic & 21 & $21 / 21(100 \%)$ & $6 / 21(28.6 \%)$ & 6 & $6 / 6(100 \%)$ & $0 / 6(0 \%)$ \\
\hline 65 & PDFontDescriptor_isSerif & 21 & $21 / 21(100 \%)$ & $6 / 21(28.6 \%)$ & 6 & $6 / 6(100 \%)$ & $0 / 6(0 \%)$ \\
\hline 66 & PDExtendedGraphicsState_getSoftMask & 20 & $20 / 20(100 \%)$ & $4 / 20(20 \%)$ & 4 & $4 / 4(100 \%)$ & $0 / 4(0 \%)$ \\
\hline 67 & PDICCBased_getDefaultDecode & 16 & $16 / 16(100 \%)$ & $8 / 18(44.4 \%)$ & 8 & $8 / 8(100 \%)$ & $0 / 8(0 \%)$ \\
\hline 68 & PDCIDSystemInfo_getOrdering & 12 & $12 / 12(100 \%)$ & $1 / 12(0.08 \%)$ & 1 & $1 / 1(100 \%)$ & $0 / 1(0 \%)$ \\
\hline 69 & PDExtendedGraphicsState_getAlphaSourceFlag & 12 & $12 / 12(100 \%)$ & $1 / 12(0.08 \%)$ & 1 & $1 / 1(100 \%)$ & $0 / 1(0 \%)$ \\
\hline 70 & PDExtendedGraphicsState_getNonStrokingAlphaConstant & 12 & $12 / 12(100 \%)$ & $1 / 12(0.08 \%)$ & 1 & $1 / 1(100 \%)$ & $0 / 1(0 \%)$ \\
\hline 71 & PDExtendedGraphicsState_getStrokingAlphaConstant & 12 & $12 / 12(100 \%)$ & $1 / 12(0.08 \%)$ & 1 & $1 / 1(100 \%)$ & $0 / 1(0 \%)$ \\
\hline 72 & PDDocumentCatalog_getViewerPreferences & 8 & $8 / 8(100 \%)$ & $8 / 8(100 \%)$ & 8 & $6 / 8(75 \%)$ & $2 / 8(25 \%)$ \\
\hline 73 & PDCIDFontType2_getPath & 6 & $6 / 6(100 \%)$ & $6 / 6(100 \%)$ & 6 & $0 / 6(0 \%)$ & $6 / 6(100 \%)$ \\
\hline 74 & PDCIDFont_getCIDSystemInfo & 3 & $3 / 3(100 \%)$ & $1 / 3(33.3 \%)$ & 1 & $0 / 1(0 \%)$ & $1 / 1(100 \%)$ \\
\hline 75 & PDCIDSystemInfo_getRegistry & 3 & $3 / 3(100 \%)$ & $1 / 3(33.3 \%)$ & 1 & $1 / 1(100 \%)$ & $0 / 1(0 \%)$ \\
\hline & PDFBOX_TOTAL & 121175 & $57563 / 121175(47.5 \%)$ & $13851 / 57563(24 \%)$ & 13851 & 13614 / $13851(98.2 \%)$ & 237 / $13851(1.8 \%)$ \\
\hline 76 & Skulmpl_getCurrency & 415 & $415 / 415(100 \%)$ & $128 / 415(30.8 \%)$ & 128 & $128 / 128(100 \%)$ & $0 / 128(0 \%)$ \\
\hline 77 & SkuImpl_getName & 394 & 394 / $394(100 \%)$ & $182 / 394(46.1 \%)$ & 182 & $84 / 182(46.1 \%)$ & 98 / $182(53.8 \%)$ \\
\hline 78 & SkuImpl_isTaxable & 24 & $24 / 24(100 \%)$ & $12 / 24(50 \%)$ & 12 & $12 / 12(100 \%)$ & $0 / 12(0 \%)$ \\
\hline 79 & OrderImpl_finalizeItemPrices & 6 & $6 / 6(100 \%)$ & $6 / 6(100 \%)$ & 6 & $6 / 6(100 \%)$ & $0 / 6(0 \%)$ \\
\hline 80 & OrderImpl_getHasOrderAdjustments & 6 & $6 / 6(100 \%)$ & $6 / 6(100 \%)$ & 6 & $6 / 6(100 \%)$ & $0 / 6(0 \%)$ \\
\hline 81 & DynamicSkuPrices_getPrice & 4 & $4 / 4(100 \%)$ & $2 / 4(50 \%)$ & 2 & $2 / 2(100 \%)$ & $0 / 2(0 \%)$ \\
\hline 82 & FixedPrice...Provider_canCalculateCostForFulfillmentGroup & 4 & $4 / 4(100 \%)$ & $2 / 4(50 \%)$ & 2 & $2 / 2(100 \%)$ & $0 / 2(0 \%)$ \\
\hline 83 & SkuImpl_getBaseRetailPrice & 4 & $4 / 4(100 \%)$ & $4 / 4(100 \%)$ & 4 & $0 / 4(0 \%)$ & $4 / 4(100 \%)$ \\
\hline 84 & Skulmpl_getBaseSalePrice & 4 & $4 / 4(100 \%)$ & $4 / 4(100 \%)$ & 4 & $4 / 4(100 \%)$ & $0 / 4(0 \%)$ \\
\hline 85 & Skulmpl_getPriceData & 4 & $4 / 4(100 \%)$ & $4 / 4(100 \%)$ & 4 & $0 / 4(0 \%)$ & $4 / 4(100 \%)$ \\
\hline 86 & FixedPriceFulfillmentOptionImpl_getPrice & 2 & $2 / 2(100 \%)$ & $1 / 2(50 \%)$ & 1 & $0 / 1(0 \%)$ & $1 / 1(100 \%)$ \\
\hline & BROADLEAF_TOTAL & 867 & $867 / 867(100 \%)$ & $351 / 867(40.5 \%)$ & 351 & $244 / 351(69.5 \%)$ & $107 / 351(30.5 \%)$ \\
\hline & TOTAL & 122194 & $\mathbf{5 8 5 4 0} / 122194(47.9 \%)$ & 14222 / $58540(24.3 \%)$ & 14222 & 13878 / $14222(97.6 \%)$ & 344 / $14222(2.4 \%)$ \\
\hline
\end{tabular}


1 public boolean isfill() \{

2 return this $==$ FILL $\|$ this == FILL_STROKE || this == FILL_CLIP $3\}$ || this == FILL_STROKE_CLIP ;

Listing 6: A target method called isFill in PDFBox

become effective target methods for PANKTI. These methods are included in rows 30 to 75 in Table III. From the 121, 175 total invocations of these target methods in production, which occur throughout the course of our experiment, we collect 57,563 production object profiles.

The collected object profiles amount to $5.5 \mathrm{~GB}$ of disk space. We notice that for 19 of the 46 target methods, PANKTI does not collect all the object profiles. This means that the size threshold of $200 \mathrm{MB}$ is encountered for these 19 methods. The distribution of the sizes of the collected object profiles is illustrated in Figure 5. From the figure, we see that most of the collected object profiles for PDFBox range from 0 to $10 \mathrm{MB}$ in size. The smallest object profile we collect is 158 bytes in size, with the largest one being nearly $55 \mathrm{MB}$. Larger object profiles correspond to complex objects with many levels of nesting, the maximum level of nesting we observe in a collected object profile is 36. As explained in subsubsection II-D3, we limit the total storage size for object profiles; to trade between the value of the collected profiles and the performance of PANKTI. In the extreme cases, such as those in rows 31,33 , and 34 in Table III, PANKTI must store only a fraction of the profiles.

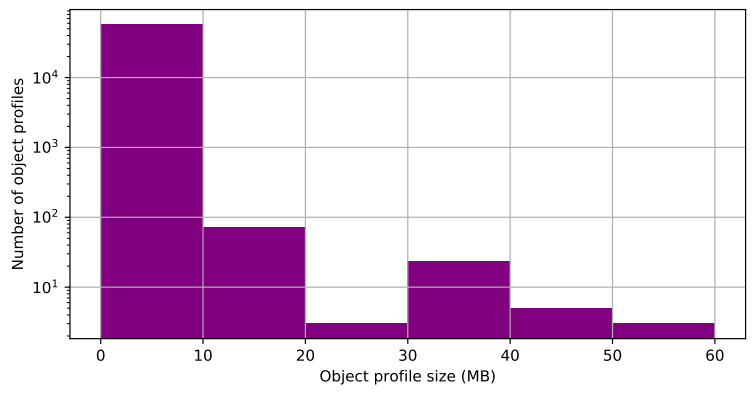

Fig. 5: The distribution of the collected object profile sizes for PDFBox over 57, 563 profiles from 46 methods

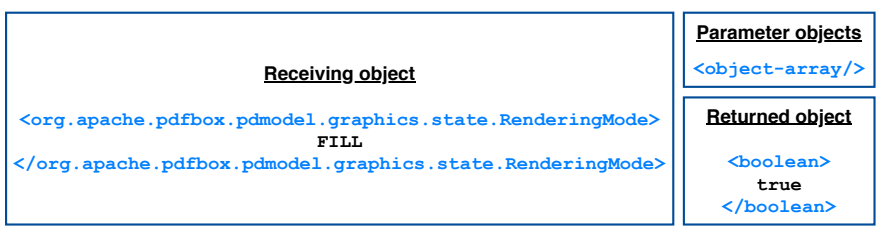

Fig. 6: A production object profile for the method isFill() in PDFBox

The set of 57,563 object profiles includes 13,851 profiles that are unique. Per the algorithm presented in subsection II-E, PANKTI generates one test method corresponding to each of these profiles. The number of generated tests ranges between a minimum of 1 for 7 target methods to a maximum of 11,730 for 1 target method. In total, we execute 13,851 differential unit tests generated by PANKTI. 13, $614(98.3 \%)$ of these tests pass while 237 tests fail. We discuss the reasons for the failure of the generated tests in subsection V-A.
1 aTest

2 public void testIsFill() \{

3 String receivingobjectStr $=$

"<org. apache, pdfbox, pdmodel, graphics, state. RenderingMode

FILL</org. apache.pdfbox. pdmodel.graphics.state.RenderingMode>";

6 RenderingMode receiving0bject $=$

deserialize0bject (receiving0bjectStr);

7 assertEquals(true, receiving0bject.isfill());

Listing 7: A generated differential unit test for the isFill method

Thanks to these generated tests, 28 of the 46 target methods $(60.9 \%)$ switch from being pseudo-tested to welltested. For example, the method called isfill in the class RenderingMode (row 30 in Table III) is one method that becomes well-tested after the addition of a PANKTI-generated test to the test suite of PDFBox.

Let us now discuss the case of the heavily-invoked method isFill, shown in Listing 6. It takes no parameter, and returns a boolean value, depending on certain values of the rendering mode for a PDF document. It is invoked 30,944 times by our production workload. We collect all 30,944 object profiles for this method, of 158 bytes each. Since all these object profiles are the same, shown in Figure 6, PANKTI generates one single test method for it. Its status upgrades from pseudo-tested to well-tested with the addition of this new test. The generated test is shown in Listing 7.

We formatted the generated test to be consistent with the structure of test methods in the PDFBox codebase, and suggested it to the developers of PDFBox in the form of a pull request ${ }^{12}$ on GitHub. The pull request was accepted by a developer, and the new test case is now part of the test suite for PDFBox. This indicates that PANKTI can capture relevant information in production and turn them into valid test inputs and oracles.

\section{Highlight from the PDFBox Experiment}

With a PDF manipulation workload for PDFBox, PANKTI generates differential unit tests that improve the testing of 28 out of 46 target pseudo-tested methods. This case study shows that the PANKTI monitoring and test generation pipeline scales to thousands of method invocations and object profiles. Furthermore, this case study validates the design decision of thresholding the number and size of collected object profiles.

\section{Case Study 3: Broadleaf}

Descartes finds 32 pseudo-tested methods when running the original test suite of the Broadleaf e-commerce application. Next, we deploy Broadleaf together with PANKTI and execute it with the typical e-commerce workload described in subsection III-B. PANKTI finds that 11 out of the 32 pseudo-tested methods are executed by our workload. These 11 effective target methods are shown in rows 76 to 86 in Table III. They are invoked 867 times during the experiment.

\footnotetext{
${ }^{12}$ https://github.com/apache/pdfbox/pull/88
} 
1 aTest

2 public void testGetHasOrderAdjustments() \{

3 File fileReceiving = new File("receiving. $x m l$ "):

4 OrderImpl receiving0bj = deserialize0bject (fileReceiving);

5 assertEquals(false, receiving0bj.getHas0rderAdjustments()):

$6\}$

Listing 8: A generated differential unit test for the getHasOrderAdjustments method

The size threshold of $200 \mathrm{MB}$ is not encountered for any of these methods. We observe that target methods are invoked throughout the duration of the experiment. Consequently, the object profiles for all invocations are successfully serialized to files by PANKTI. During the test generation phase, PANKTI identifies 351 unique object profiles from the collected ones. All of the unique object profiles are transformed into unit tests without any exception.

When executing the 351 generated differential unit tests, $244(69.5 \%)$ of them pass, while the other $107(30.5 \%)$ fail. There are several reasons why some generated tests may fail, which will be discussed in subsection $\mathrm{V}$-A. The passing tests improve the test quality for 6 out of the 11 (54.5\%) target methods. This means that PANKTI generates tests that better specify the behavior of these 6 methods.

Let us take row 80 in Table III as an example. In Broadleaf's original test suite, the method getHas0rderAdjustments in class OrderImpl is pseudo-tested. If the body of this method is replaced by return true; or return false; none of the 6 test cases that reach it fail, meaning that even though the method is covered, its correct behavior is not specified by the existing tests. In production, PANKTI collects 6 unique object profiles for this method. Listing 8 presents one of the test cases generated according to the object profiles. In the generated test, the receiving object is deserialized from the profile (since the profile is large, the object profile is read from a file for the sake of test readability) (line 4). According to the collected production data, the method invocation is expected to return false. Thus assertEquals (false, ....) is generated to verify the actual return value of method getHas0rderAdjustments (line 5 ). After the addition of this newly generated differential unit test to the test suite of Broadleaf, Descartes does not detect getHas0rderAdjustments as a pseudo-tested method anymore. This implies that the values collected by observing the interactions of a real user with the website can contribute to the improvement of the overall quality of the test suite of Broadleaf.

\begin{tabular}{l}
\hline Highlight from the Broadleaf Experiment \\
\hline PANKTI collects 351 unique object profiles while running \\
the production workload on the e-commerce application \\
Broadleaf, and successfully generates a test case for each \\
profile. In total, 244 test cases improve the test thoroughness \\
for 6 out of the 11 target pseudo-tested methods. Overall, this \\
case study shows that PANKTI works well in a typical web, \\
HTTP-based architecture which is representative of many \\
enterprise applications.
\end{tabular}

\section{E. Experimental Results with System Tests}

Table IV summarizes the results of the assessment of PANKTI's ability to trace the execution of system tests in order to generate differential unit tests, per the protocol of subsection III-C. For each of the three study subjects. the number of system tests we identify is given in the column \#SYSTEM_TESTS. \#EFF_TARGET represents the number of target pseudo-tested methods invoked during the execution of these system tests. The number of differential unit tests generated using the object profiles collected by PANKTI is signified by \#PANKTI_TESTS. Of these, the tests that pass are represented as \#PASSING, and the ones that do not, as \#FAILING. In column PANKTI_STATUS we provide the status of the invoked target methods after adding the PANKTIgenerated differential unit tests to the test suite of the project. They either remain pseudo-tested, or become well-tested.

The table indicates that there are 11, 150, and 6 system tests in Jicofo, PDFBox, and Broadleaf, respectively. PANKTI instruments 29, 138, and 32 pseudo-tested methods in these projects. Of these instrumented methods, 29, 111, and 26 are invoked during the execution of the system tests, and are the effective targets for test generation. PANKTI successfully generates 20, 18, 291, and 692 differential unit tests for these target methods. Of these tests, $100 \%, 96.5 \%$, and $98.8 \%$ pass. As a result of the tests generated by PANKTI, $62 \%$ (18 of 29), $59.5 \%$ (66 of 111), and $46.1 \%$ (12 of 26 ) of targets in Jicofo, PDFBox, and Broadleaf are no longer pseudo-tested.

We note that the set of methods invoked during our experiments with production workloads differs from the ones invoked with system tests, i.e., for the same \#TARGET, \#EFF_TARGET is different for the two experiments. We observe that the production workload for PDFBox invokes 2 methods which are never reached by system tests. One of these methods becomes well-tested as a result of the tests generated by PANKTI. For Broadleaf, the production workload reaches 3 methods not invoked at all by system tests, of which 2 methods become well-tested due to the addition of the new tests. For Jicofo, both the production workload and the system tests cover the same set of methods. These observations confirm that system tests can miss some behavior exercised in production [9]. Consequently, a production workload is an essential complement to system tests for test improvement, as it provides additional test generation targets for PANKTI.

In total, PANKTI generates tests for 166 out of the 199 instrumented pseudo-tested methods, using 167 system tests across the three study subjects. 96 of these methods $(57.8 \%)$ become well-tested as a result of the tests automatically generated by PANKTI. This experiment demonstrates that PANKTI can generate differential unit tests from system tests.

\section{Discussion}

We now discuss the key aspects of PANKTI.

\section{A. When PANKTI-generated Tests Fail}

From Table III, we observe that a total of 344 PANKTIgenerated unit tests fail (2.4\% of the generated tests). We manually analyze a random sample of them to understand the 
TABLE IV: Experimental results from executing system tests of the study subjects used to evaluate PANKTI

\begin{tabular}{lrrrrrr}
\hline PROJECT & \#SYSTEM_TESTS & \#EFF_TARGET & \#PANKTI_TESTS & \#PASSING & \#FAILING & PANKTI_STATUS \\
\hline $\begin{array}{l}\text { Jicofo } \\
\text { PDFBox }\end{array}$ & 11 & $\mathbf{2 9} / 29$ & 20 & $\mathbf{2 0} / 20$ & $\mathbf{0} / 20$ & $\begin{array}{r}\text { well-tested: } \mathbf{1 8} / 29 \\
\text { pseudo-tested: } \mathbf{1 1} / 29\end{array}$ \\
\hline Broadleaf & 6 & $\mathbf{1 1 1} / 138$ & 18,291 & $\mathbf{1 7 , 6 5 5} / 18,291$ & $\mathbf{6 3 6} / 18,291$ & $\begin{array}{r}\text { well-tested: } \mathbf{6 6} / 111 \\
\text { pseudo-tested: } \mathbf{4 5} / 111\end{array}$ \\
\hline TOTAL & 167 & $\mathbf{2 6} / 32$ & 692 & $\mathbf{6 8 4} / 692$ & $\mathbf{8} / 692$ & $\begin{array}{r}\text { well-tested: } \mathbf{1 2} / 26 \\
\text { pseudo-tested: } \mathbf{1 4} / 26\end{array}$ \\
\hline
\end{tabular}

causes. First, we find that no test generated for methods that return a primitive type, an array of a primitive type, a wrapper object for a primitive type, or an object of an enum type fail. For the tests that fail in PDFBox and Broadleaf, all methods return complex objects. Our manual analysis reveals four main reasons for a failure in a PANKTI-generated test.

Comparison with the equals method can fail for arbitrary objects. An assertEquals JUnit assertion internally invokes the equals method on the two objects being compared. According to the default implementation of equals, a value of true is returned for two objects if they have the same reference or address in memory. To perform a deep comparison on the two objects, the behavior of equals must be overridden [39]. As this is not guaranteed for the arbitrary objects we serialize during our experiments, the equality assertions, even for two objects that are otherwise internally identical, are prone to failure if the equals method is not, or partially, implemented. This is the case for the target methods of Broadleaf in row 85, and PDFBox in rows 51, 72, and 73 of Table III.

An external component or a cache is not available during testing. Tests can fail due to the unavailability of external components at testing time (while being available in production). For example, the method getName in Broadleaf (row 77 in Table III) returns the name of a product as a java.lang.String object. When PANKTI collects object profiles in the production environment, the method getName returns the name of a product provided through requests to an external translation service. However, during test execution, the method getName always returns the default English name of a product because the translation service is not activated. Tests may also fail due to the unavailability of internal caches. The target methods for PDFBox in rows 37, 44, 45, 46, and 50 in Table III access objects from in-memory caches implemented using java.lang.ref. SoftReference, which are not available in the testing environment, causing the assertions to fail.

The target methods return objects containing transient fields. The objects being returned by the target methods for PDFBox in rows 33, 34, 35, 53, and 73 of Table III contain transient fields which are not serialized, by definition. Examples of such objects include java.awt.geom. GeneralPath and java.util.IdentityHashMap. This leads to failing assertions during the comparison of the returned object during the method invocation in the generated test.

The class declaring the target method overrides the se- rialization behavior. XStream has different converters for different kinds of classes. If a class defines its own serialization behavior, for example, by implementing the java.io.Externalizable interface, XStream follows it. If the serialization or the deserialization behavior is not well designed, it can possibly lead to an assertion failure. The tests for method getBaseRetailPrice in Broadleaf (row 83 in Table III) fail for this reason. The method returns an object whose type is Money. Money customizes its deserialization such that a java.math.BigDecimal field is converted from a float value. However, this changes the scale of the BigDecimal, which makes the assertion fail in the generated test.

\section{B. Cases where the Test Suite is not Improved}

From Table III, we see that PANKTI generates at least one differential unit test that passes for 15 target methods, yet their status does not upgrade from pseudo-tested to tested. We analyze these cases manually. For those 15 target methods, PANKTI generates valid differential unit tests that check the behavior of these target methods when they return null, which is still a weak assertion.

We run Descartes, to determine the status of the target methods after adding the PANKTI-generated tests. For each of these 15 methods, Descartes performs one transformation on the methods: replace the body with return null; . The PANKTI-generated tests do not distinguish this transformation from the normal behavior of the method, and thus do not fail. This is a corner case of the default test adequacy criterion of PANKTI based on pseudo-tested methods. PANKTI generates valid, relevant test cases, yet the extreme case where Descartes generates a single variant goes unnoticed.

\section{Overhead of Generating Tests from Production Workloads}

This section reports a thorough performance evaluation of the deployment overhead of PANKTI for the three study subjects. For PDFBox, we compute the average CPU and memory utilization of 10 normal executions of the workload defined in subsubsection III-B2, 10 executions of this workload which we monitor with Glowroot only, and 10 executions with PANKTI fully attached (Glowroot, together with the instrumentation and serialization). We find that CPU usage is $3.7 \%$ during normal execution, and $21 \%$ with Glowroot attached. We also observe that attaching PANKTI does not introduce any additional increase in CPU usage. Memory usage is $65 \mathrm{MB}$ during 
normal execution, $180 \mathrm{MB}$ while execution of the workload with Glowroot, and $557 \mathrm{MB}$ after attaching PANKTI.

Overhead is an important aspect for interactive clientfacing applications such as Jitsi/Jicofo (videoconference) and Broadleaf (e-commerce), because it may degrade user experience. For a Jitsi videoconference, without PANKTI, we observe an average CPU and memory usage of $0.1 \%$ and 338 $\mathrm{MB}$, respectively. When attaching PANKTI, we observe $5.5 \%$ CPU usage and $902 \mathrm{MB}$ memory. These values drop to $0.4 \%$ and $688 \mathrm{MB}$, respectively, when ignoring the target methods that raise exceptions related to serialization (discussed in subsection IV-B). Packet loss rate remains stable at zero for all experiments, implying that users do not experience any side effects on video or audio quality from lost packets due to PANKTI. For Broadleaf, the average CPU and memory usage during normal execution are $16.4 \%$ and $1081 \mathrm{MB}$, respectively, and $41.8 \%$ and $1130 \mathrm{MB}$ with PANKTI attached. To determine the impact on user experience, we measure the average response time for HTTP requests with apache-utils $s^{13}$ and find that it increases by an average of 185 milliseconds. The results from these experiments can also be found at https://github.com/castor-software/pankti-experiments.

PANKTI builds on top of Glowroot, a widely-adopted, stateof-the-art monitoring solution for Java projects. Glowroot constitutes a significant part of the overall PANKTI overhead, yet, it is still acceptable for a standard server running in a real production scenario [40], and this computation price is paid by many applications in the world, in order to access the state-of-the-art monitoring provided by Glowroot. It is also important to note that these values of overhead are subject to change depending on the workload, the set of target methods, and the threshold for object profile collection, all of which are completely configurable factors.

\section{Coverage Change with PANKTI}

The code coverage ratio of the three case studies discussed in section III slightly increases after adding the tests generated by PANKTI to the original test suites. For Jicofo, the PANKTI differential unit tests cover five new lines of code and six new branches. For PDFBox, the addition of the new tests results in the coverage of four more methods in the application. For Broadleaf, the new tests increase test suite coverage by one line and two branches.

This outcome is to be expected because the test adequacy criterion used in our evaluation is pseudo-tested methods (see subsection III-D). Recall that, by construction, these target methods are already covered by the original test suite of the three projects. For this reason, the only coverage increase may happen in a few uncovered branches in covered methods. To this extent, code coverage can be considered as irrelevant. Instead, we fully focus on the quality of the oracle, and assess the improvement provided by PANKTI tests with regards to the oracle: still pseudo-tested or not, after adding the PANKTI tests. An interesting area of future work would be to study the impact of PANKTI-generated tests on coverage, when the targets for test generation are also selected based on coverage.

${ }^{13}$ https://httpd.apache.org/docs/2.4/programs/ab.html

\section{E. Privacy Implications}

As regulations, such as the General Data Protection Regulation (GDPR) in the European Union, gain traction, stricter policies are being enforced to address issues concerning the storage and processing of user data. One technical challenge induced by these regulations consists in finding a good balance between effective monitoring of software systems in production, and secure and lawful handling of user data [41]. PANKTI has privacy implications, since the production workload and object profiles may contain sensitive information, such as user names and passwords. We mitigate a part of this risk with one explicit input that specifies a selection of target methods to be instrumented: the list of target methods can be audited and adjusted by developers first, according to privacy and regulations. In this way, PANKTI would focus its instrumentation and monitoring on non-sensitive methods only. Without involving developers, it is also possible to protect users' privacy with the state-of-the-art of automated privacy-preservation techniques [42], [43]. Overall, similar to related work on production monitoring, PANKTI has to trade-off between its capabilities and compliance with data protection laws [44].

\section{F. Structure and Readability of the PANKTI Differential Unit Tests}

Readability is an important aspect of automatically generated tests [45], [46]. A test produced by an automated generation tool is more likely to be seamlessly integrated into an existing test suite if developers can understand it. To this end, we engineer PANKTI to ensure that generated tests are targeted and focused. For our three study subjects, each of the tests generated by PANKTI contains a clear test intention, represented in the form of a single invocation of the target method with a unique input, and a single assertion on equality of the output of the invocation and the oracle. This systematic template and precise intention ensure that these tests are inherently well-structured and easy to understand. The qualitative feedback obtained on the PANKTI test given as pull-request (see subsection IV-C) tends to validate this.

As described in subsection II-E, the tests generated by PANKTI rely on production object profiles which are deserialized from the XML format during the execution of the test. Despite the well-defined structure of the tests themselves, we appreciate that these profiles can often be lengthy and potentially challenging to comprehend. As future work, we plan to conduct user studies involving developers in order to analyze the readability and debuggability [47] of the tests generated by PANKTI.

\section{G. Threats to Validity}

The main internal threat to validity comes from the libraries that PANKTI uses for the implementation. In the current version, XStream is used as the serialization and deserialization library. Though XStream is the state-of-the-art tool for object serialization, it sometimes fails for some special types of objects if it is not customized. For example, objects that are based on threads or thread data can not be serialized 
by default ${ }^{14}$. Yet, a serialization failure rarely happens for our experiments: we observe this only once in Jicofo. The workaround is engineering: it is possible to improve the ability of XStream's serialization by registering customized object converters.

The threat to external validity is related to the breadth of considered application domains. We mitigate the external threat to validity by evaluating PANKTI with three Java projects, as discussed in section III. The main strength is that they are real-world applications that cover different production workloads and diverse production environments. We look forward to applying PANKTI with our industry partners to further improve external validity.

\section{RELATED WORK}

PANKTI contributes to the field of automatic generation and automatic improvement of test suites. The generation of relevant test inputs [48] is a key challenge in both these domains, which has been addressed through symbolic [49], model-based [50], or search-based techniques [51]. The novelty of PANKTI is twofold: first, to collect data in production and automatically turn them into test inputs; second, to target specific parts of the application code that are weakly-tested and of interest for the developers, in order to generate test cases that are valuable for the quality of the test suite.

The closest work to PANKTI is a recently developed tool called Replica, by Wang and Orso [52]. It traces production behavior as a sequence of method calls and uses these traces to spot behaviors triggered in production but not covered by the test suite. Replica then uses a guided symbolic execution of the program to "mimic" this behavior and to generate inputs for untested behavior. The primary difference with PANKTI is that we generate test oracles from the actual observations made in production, and not from symbolic execution. PANKTI also collects a different type of trace compared to Replica: object profiles instead of sequences of method calls. Focusing on object profiles, we can generate test data that recreate production conditions.

\section{A. Generating tests from execution traces}

Thummalapenta et al. [53] mine program execution traces, which include method calls, arguments, and return values, to generate parameterized unit tests implemented as regression tests with Pex [54]. Marchetto et al. [55] define a methodology to generate Selenium tests from event logs for web applications. Sampath et al. [56] apply incremental concept analysis to cluster similar test cases, generated from user-session based testing of web applications. Several works generate test cases that reproduce failures [57], [58], [29]. In particular, Artzi et al. propose ReCrash ${ }^{15}$ to turn runtime observations into unit tests that reproduce failures [29]. ReCrash works in a similar fashion as PANKTI in that it serializes object states observed during execution. However, unlike PANKTI, its goal is not to generate tests for target methods that need better

\footnotetext{
${ }^{14} \mathrm{https} / / / \mathrm{X}$-stream.github.io/faq.html\#Serialization_types

${ }^{15}$ http://groups.csail.mit.edu/pag/reCrash/
}

testing, but to generate tests that raise the same exceptions as observed in production. In particular, ReCrash has no feature to create derived oracles. More recently, Utting et al. [59] apply machine learning to user and test execution traces in order to identify test inadequacies and generate new tests for usage scenarios that are missing from the test suite. Krrikava and Vitek [26] record function calls, including arguments, in execution traces of software packages developed in the $\mathrm{R}$ language, and extract unit tests from them with the goal of improving coverage. Similar to these studies, PANKTI relies on the observation of an executing application for test generation. Compared to these tools, the key novelty of PANKTI is the execution representation using object profiles, which has never been proposed so far. Moreover, PANKTI allows developers to choose which methods to target for test generation.

\section{B. Test suite improvement}

Danglot et al. [38] and Baudry et al. [60] propose to transform existing test methods into new ones that globally improve the coverage and the mutation score of the test suite [61]. Tillman and Schulte [62] suggest the use of symbolic execution to generalize traditional unit tests into parameterized unit tests and instantiating these PUTs to obtain concrete tests for higher test coverage. Saff et al. [36] develop a test refactoring technique to automatically generate unit tests from bulky system tests. Harder et al. [8] compute the operational difference based on the behavior of a program observed during execution in semantic terms with Daikon [63] to keep only those test cases that add to the abstraction. This results in a test suite that is minimal but better at detecting faults than a suite with high coverage. Chen et al. [64] address the issues related to behavioral backward incompatibilities (BBIs), and propose the early detection of BBIs in libraries across their client projects by prioritizing and executing the clients' tests. The goal of these studies, like ours, is to improve the test quality of the project. The key differences are that PANKTI bases its test input and derived oracle generation process on observations collected in production, and that it targets test generation on a subset of methods that the developers consider as relevant for test improvement.

\section{Handling the oracle problem}

The oracle problem in software testing refers to the identification of the desired behavior of a program unit [65]. In their survey, Barr et al. [2] investigate four aspects in the literature that address the oracle problem, namely specified, derived, and implicit oracles, and the lack of automated test oracles. Gay et al. [66] combine monitoring the execution of test cases, with mutation testing, in order to automatically select oracle data. Bertolino et al. [67] execute tests in-vivo, as an application executes, effectively leveraging production data as oracles and allowing the discovery of corner-cases that may otherwise be rare in the testing environment. The oracle can be automated, taking the domain into account. For instance, the ADVISOR tool of Genç et al. [68] automates the test oracle in systems with a graphical/visual output, which can otherwise be very inaccurate due to contextual differences between an 
output image and a reference image. PANKTI approaches the oracle problem by automatically synthesizing derived oracles from production observations, which is novel to the best our knowledge.

Our work is different from the technique of Elbaum et al. [14], who also generate derived oracles: they use system tests whereas PANKTI uses production workloads; their approach does not allow the set of target methods to be configured according to a developer-defined criterion. Moreover, PANKTI can generate tests for methods that are never tested, while the technique of Elbaum et al. generates test cases exclusively for methods that are covered by system-level tests

\section{Capture and replay}

Joshi and Orso [69] present a capture and replay tool, and propose potential applications, including the generation of regression tests. Their tool captures selectively at the boundary of a subset of the application under study as it executes. Steven et al. [70] design a tool called JRapture that captures the sequence of interactions between an executing Java program and components on the host system such as files, or events on graphical user interfaces. These sequences can then be replayed for observation-based testing. GenUTest [28] is a capture and replay tool which logs method calls and the sequence of their occurrence, in a medium-sized, executing instrumented program. The arguments passed to and values returned from methods are serialized into $\log$, which are utilized for the generation of unit tests and mocks for methods, including test assertions. Saieva et al. [71] generate ad-hoc tests that replay recorded user execution traces in order to test candidate patches for critical security bugs. The uniqueness of PANKTI is that object profiles are captured not for the purpose of replaying the same sequence of operations on the system, but for specifying the behavior of target methods, through focused differential unit tests, with derived test oracles. This is in contrast to capture and replay systems, which typically lack oracles.

\section{CONCLUSION}

This paper has introduced PANKTI, a tool that observes Java programs in production to automatically generate differential unit tests. PANKTI observes specific methods that are weaklytested according to a test adequacy criterion and introduces a novel technique based on the collection of object profiles. We have conducted experiments with three sizeable, popular, multi-domain, open-source Java projects to assess PANKTI's ability at monitoring production and at improving real-world test suites. PANKTI successfully generates differential unit tests that improve the testing of 53 of the 86 (61.6\%) methods targeted across our three study subjects. This shows that PANKTI is able to generate tests for real-world Java software.

In follow-up studies, we will broaden the scope of test improvement to other weakly-tested parts. In particular, one can focus on methods that are not reached by any existing test but executed in the field, the existence of which has been demonstrated by Wang et al. [9] and Gittens et al. [72]. Also, we wish to explore test input minimization by utilizing partial object profiles instead of the whole. Our second thread for future work consists in extending PANKTI to generate integration- or system-level tests, by considering arbitrary sequences of method invocations across different classes.

\section{REFERENCES}

[1] T. Y. Chen and R. Merkel, "An upper bound on software testing effectiveness," ACM Transactions on Software Engineering and Methodology (TOSEM), vol. 17, no. 3, pp. 1-27, 2008.

[2] E. T. Barr, M. Harman, P. McMinn, M. Shahbaz, and S. Yoo, "The oracle problem in software testing: A survey," IEEE transactions on software engineering, vol. 41, no. 5, pp. 507-525, 2014.

[3] G. Catolino, F. Palomba, A. Zaidman, and F. Ferrucci, "How the experience of development teams relates to assertion density of test classes," in 2019 IEEE International Conference on Software Maintenance and Evolution, ICSME 2019, Cleveland, OH, USA, September 29 - October 4, 2019, pp. 223-234, IEEE, 2019.

[4] P. S. Kochhar, D. Lo, J. Lawall, and N. Nagappan, "Code coverage and postrelease defects: A large-scale study on open source projects," IEEE Transactions on Reliability, vol. 66, no. 4, pp. 1213-1228, 2017.

[5] G. Grano, C. De Iaco, F. Palomba, and H. C. Gall, "Pizza versus pinsa: On the perception and measurability of unit test code quality," in Proc. of the International Conference on Software Maintenance and Evolution (ICSME), pp. 336-347, 2020.

[6] B. Danglot, O. Vera-Perez, Z. Yu, A. Zaidman, M. Monperrus, and B. Baudry, "A snowballing literature study on test amplification," Journal of Systems and Software, vol. 157, 2019.

[7] T. Xie, "Augmenting automatically generated unit-test suites with regression oracle checking," in European Conference on Object-Oriented Programming, pp. 380-403, Springer, 2006.

[8] M. Harder, J. Mellen, and M. D. Ernst, "Improving test suites via operational abstraction," in 25th International Conference on Software Engineering, 2003. Proceedings., pp. 60-71, IEEE, 2003.

[9] Q. Wang, Y. Brun, and A. Orso, "Behavioral execution comparison: Are tests representative of field behavior?," in 2017 IEEE International Conference on Software Testing, Verification and Validation (ICST), pp. 321-332, IEEE, 2017.

[10] M. Gligoric, A. Groce, C. Zhang, R. Sharma, M. A. Alipour, and D. Marinov, "Comparing non-adequate test suites using coverage criteria," in Proceedings of the 2013 International Symposium on Software Testing and Analysis, pp. 302-313, 2013.

[11] M. Hilton, J. Bell, and D. Marinov, "A large-scale study of test coverage evolution," in Proc. of the International Conference on Automated Software Engineering, ASE, pp. 53-63, 2018.

[12] G. Petrović and M. Ivanković, "State of mutation testing at google," in Proceedings of the 40th international conference on software engineering: Software engineering in practice, pp. 163-171, 2018.

[13] Y. Ma, J. Offutt, and Y. R. Kwon, "Mujava: a mutation system for java," in Proc. of the International Conference on Software Engineering (ICSE), pp. 827-830, 2006.

[14] S. Elbaum, H. N. Chin, M. B. Dwyer, and J. Dokulil, "Carving differential unit test cases from system test cases," in Proceedings of the 14th ACM SIGSOFT international symposium on Foundations of software engineering, pp. 253-264, 2006.

[15] R. Niedermayr, E. Juergens, and S. Wagner, "Will my tests tell me if i break this code?," in 2016 IEEE/ACM International Workshop on Continuous Software Evolution and Delivery (CSED), pp. 23-29, IEEE, 2016.

[16] L. Inozemtseva and R. Holmes, "Coverage is not strongly correlated with test suite effectiveness," in Proceedings of the 36th international conference on software engineering, pp. 435-445, 2014.

[17] Y. Zhang and A. Mesbah, "Assertions are strongly correlated with test suite effectiveness," in Proceedings of the 2015 10th Joint Meeting on Foundations of Software Engineering, pp. 214-224, 2015.

[18] L. Zhang and M. Monperrus, "Tripleagent: Monitoring, perturbation and failure-obliviousness for automated resilience improvement in java applications," in Proceedings of the IEEE International Symposium on Software Reliability Engineering, 2019.

[19] B. Danglot, M. Monperrus, W. Rudametkin, and B. Baudry, "An approach and benchmark to detect behavioral changes of commits in continuous integration," Empirical Software Engineering, vol. 25, no. 4, pp. 2379-2415, 2020. 
[20] Y. T. Chen, R. Gopinath, A. Tadakamalla, M. D. Ernst, R. Holmes, G. Fraser, P. Ammann, and R. Just, "Revisiting the relationship between fault detection, test adequacy criteria, and test set size," in 2020 35th IEEE/ACM International Conference on Automated Software Engineering (ASE), pp. 237-249, IEEE, 2020.

[21] O. L. Vera-Pérez, B. Danglot, M. Monperrus, and B. Baudry, "A comprehensive study of pseudo-tested methods," Empirical Software Engineering, vol. 24, no. 3, pp. 1195-1225, 2019.

[22] S. Butler, J. Gamalielsson, B. Lundell, C. Brax, A. Mattsson, T. Gustavsson, J. Feist, and E. Lönroth, "Maintaining interoperability in open source software: A case study of the apache pdfbox project," Journal of Systems and Software, vol. 159, p. 110452, 2020.

[23] D. Schuler and A. Zeller, "Assessing oracle quality with checked coverage," in 2011 Fourth IEEE International Conference on Software Testing, Verification and Validation, pp. 90-99, 2011.

[24] E. Costlow, "The State of Java Serialization." https://www.infoq.com/ articles/java-serialization-aug18/ (accessed 2020-10-14).

[25] K. Maeda, "Performance evaluation of object serialization libraries in xml, json and binary formats," in 2012 Second International Conference on Digital Information and Communication Technology and it's Applications (DICTAP), pp. 177-182, 2012.

[26] F. Křikava and J. Vitek, "Tests from traces: automated unit test extraction for r," in Proceedings of the 27th ACM SIGSOFT International Symposium on Software Testing and Analysis, pp. 232-241, 2018.

[27] J. Bell, O. Legunsen, M. Hilton, L. Eloussi, T. Yung, and D. Marinov, "Deflaker: Automatically detecting flaky tests," in 2018 IEEE/ACM 40th International Conference on Software Engineering (ICSE), pp. 433-444, IEEE, 2018.

[28] B. Pasternak, S. Tyszberowicz, and A. Yehudai, "Genutest: a unit test and mock aspect generation tool," International journal on software tools for technology transfer, vol. 11, no. 4, p. 273, 2009.

[29] S. Artzi, S. Kim, and M. D. Ernst, "Recrash: Making software failures reproducible by preserving object states," in European conference on object-oriented programming, pp. 542-565, Springer, 2008.

[30] R. Pawlak, M. Monperrus, N. Petitprez, C. Noguera, and L. Seinturier "Spoon: A Library for Implementing Analyses and Transformations of Java Source Code," Software: Practice and Experience, vol. 46, pp. 1155-1179, 2015. update for oadoi on Nov 022018.

[31] B. Flyvbjerg, "Five misunderstandings about case-study research," Qualitative inquiry, vol. 12, no. 2, pp. 219-245, 2006.

[32] S. Garfinkel, P. Farrell, V. Roussev, and G. Dinolt, "Bringing science to digital forensics with standardized forensic corpora," digital investigation, vol. 6, pp. S2-S11, 2009.

[33] T. Kuchta, T. Lutellier, E. Wong, L. Tan, and C. Cadar, "On the correctness of electronic documents: studying, finding, and localizing inconsistency bugs in pdf readers and files," Empirical Software Engineering, vol. 23, no. 6, pp. 3187-3220, 2018.

[34] S. Messaoudi, D. Shin, A. Panichella, D. Bianculli, and L. Briand, "Log-based slicing for system-level test cases," in 2021 ACM SIGSOFT International Symposium on Software Testing and Analysis (ISSTA), 2021.

[35] C. Wang, F. Pastore, A. Goknil, L. Briand, and Z. Iqbal, "Automatic generation of system test cases from use case specifications," in Proceedings of the 2015 international symposium on software testing and analysis, pp. 385-396, 2015.

[36] D. Saff, S. Artzi, J. H. Perkins, and M. D. Ernst, "Automatic test factoring for java," in Proceedings of the 20th IEEE/ACM international Conference on Automated software engineering, pp. 114-123, 2005.

[37] M. Jorde, S. Elbaum, and M. B. Dwyer, "Increasing test granularity by aggregating unit tests," in 2008 23rd IEEE/ACM International Conference on Automated Software Engineering, pp. 9-18, 2008.

[38] B. Danglot, O. L. Vera-Pérez, B. Baudry, and M. Monperrus, "Automatic test improvement with dspot: a study with ten mature open-source projects," Empirical Software Engineering, vol. 24, no. 4, pp. $2603-$ $2635,2019$.

[39] B. Weidig, "Equality and Comparison in Java: Pitfalls and Best Practices." Link to Medium post (accessed 2020-11-19).

[40] O. Cornejo, D. Briola, D. Micucci, and L. Mariani, "In-the-field monitoring of functional calls: Is it feasible?," Journal of Systems and Software, vol. 163 , p. 110523, 2020.

[41] F. Menges, T. Latzo, M. Vielberth, S. Sobola, H. C. Pöhls, B. Taubmann, J. Köstler, A. Puchta, F. Freiling, H. P. Reiser, et al., "Towards gdprcompliant data processing in modern siem systems," Computers \& Security, vol. 103, p. 102165, 2021.

[42] H. Zhang, S. Latif, R. Bassily, and A. Rountev, "Differentiallyprivate control-flow node coverage for software usage analysis," in 29th USENIX Security Symposium, USENIX Security 2020, August $12-$ 14, 2020 (S. Capkun and F. Roesner, eds.), pp. 1021-1038, USENIX Association, 2020.

[43] T. Pulls, R. Peeters, and K. Wouters, "Distributed privacy-preserving transparency logging," in Proceedings of the 12th ACM Workshop on Workshop on Privacy in the Electronic Society, WPES '13, (New York, NY, USA), p. 83-94, Association for Computing Machinery, 2013.

[44] A. Shah, V. Banakar, S. Shastri, M. Wasserman, and V. Chidambaram, "Analyzing the impact of $\{$ GDPR $\}$ on storage systems," in 11th \{USENIX\} Workshop on Hot Topics in Storage and File Systems (HotStorage 19), 2019.

[45] F. Palomba, D. Di Nucci, A. Panichella, R. Oliveto, and A. De Lucia, "On the diffusion of test smells in automatically generated test code: An empirical study," in 2016 IEEE/ACM 9th International Workshop on Search-Based Software Testing (SBST), pp. 5-14, 2016.

[46] E. Daka, J. Campos, G. Fraser, J. Dorn, and W. Weimer, "Modeling readability to improve unit tests," in Proceedings of the 2015 10th Joint Meeting on Foundations of Software Engineering, pp. 107-118, 2015.

[47] A. J. Ko and B. A. Myers, "Designing the whyline: a debugging interface for asking questions about program behavior," in Proceedings of the SIGCHI conference on Human factors in computing systems, pp. 151158,2004

[48] S. Anand, E. K. Burke, T. Y. Chen, J. Clark, M. B. Cohen, W. Grieskamp, M. Harman, M. J. Harrold, P. Mcminn, A. Bertolino, et al., "An orchestrated survey of methodologies for automated software test case generation," Journal of Systems and Software, vol. 86, no. 8, pp. 1978-2001, 2013.

[49] G. Fraser, F. Wotawa, and P. E. Ammann, "Testing with model checkers: a survey," Software Testing, Verification and Reliability, vol. 19, no. 3, pp. 215-261, 2009.

[50] M. Utting, A. Pretschner, and B. Legeard, "A taxonomy of model-based testing approaches," Software testing, verification and reliability, vol. 22, no. 5, pp. 297-312, 2012.

[51] P. McMinn, "Search-based software testing: Past, present and future," in 2011 IEEE Fourth International Conference on Software Testing, Verification and Validation Workshops, pp. 153-163, IEEE, 2011.

[52] Q. Wang and A. Orso, "Improving testing by mimicking user behavior," in 2020 IEEE International Conference on Software Maintenance and Evolution (ICSME), pp. 488-498, IEEE, 2020.

[53] S. Thummalapenta, J. De Halleux, N. Tillmann, and S. Wadsworth, "Dygen: automatic generation of high-coverage tests via mining gigabytes of dynamic traces," in International Conference on Tests and Proofs, pp. 77-93, Springer, 2010.

[54] N. Tillmann and J. De Halleux, "Pex-white box test generation for. net," in International conference on tests and proofs, pp. 134-153, Springer, 2008.

[55] A. Marchetto, P. Tonella, and F. Ricca, "State-based testing of ajax web applications," in 2008 1st International Conference on Software Testing, Verification, and Validation, pp. 121-130, IEEE, 2008.

[56] S. Sampath, S. Sprenkle, E. Gibson, L. Pollock, and A. S. Greenwald, "Applying concept analysis to user-session-based testing of web applications," IEEE Transactions on Software Engineering, vol. 33, no. 10, pp. 643-658, 2007.

[57] P. Derakhshanfar, X. Devroey, G. Perrouin, A. Zaidman, and A. van Deursen, "Search-based crash reproduction using behavioural model seeding," Softw. Test. Verification Reliab., vol. 30, no. 3, 2020.

[58] J. Xuan, X. Xie, and M. Monperrus, "Crash reproduction via test case mutation: let existing test cases help," in Proc. of the Joint Meeting on Foundations of Software Engineering, ESEC/FSE, pp. 910-913, ACM, 2015.

[59] M. Utting, B. Legeard, F. Dadeau, F. Tamagnan, and F. Bouquet, "Identifying and generating missing tests using machine learning on execution traces," in Proc. of the International Conference On Artificial Intelligence Testing, pp. 83-90, 2020.

[60] B. Baudry, F. Fleurey, J. Jézéquel, and Y. L. Traon, "Genes and bacteria for automatic test cases optimization in the .net environment," in Proc. of the Int. Symposium on Software Reliability Engineering (ISSRE), pp. 195-206, 2002.

[61] R. A. DeMillo, R. J. Lipton, and F. G. Sayward, "Hints on test data selection: Help for the practicing programmer," Computer, vol. 11, no. 4, pp. 34-41, 1978.

[62] N. Tillmann and W. Schulte, "Unit tests reloaded: Parameterized unit testing with symbolic execution," IEEE software, vol. 23, no. 4, pp. 3847, 2006.

[63] M. D. Ernst, J. H. Perkins, P. J. Guo, S. McCamant, C. Pacheco, M. S. Tschantz, and C. Xiao, "The daikon system for dynamic detection of likely invariants," Science of computer programming, vol. 69, no. 1-3, pp. 35-45, 2007. 
[64] L. Chen, F. Hassan, X. Wang, and L. Zhang, "Taming behavioral backward incompatibilities via cross-project testing and analysis," in Proceedings of the ACM/IEEE 42nd International Conference on Software Engineering, pp. 112-124, 2020.

[65] M. Staats, M. W. Whalen, and M. P. E. Heimdahl, "Programs, tests, and oracles: the foundations of testing revisited," in Proc. of the International Conference on Software Engineering (ICSE), pp. 391-400, 2011.

[66] G. Gay, M. Staats, M. W. Whalen, and M. P. E. Heimdahl, "Automated oracle data selection support," IEEE Trans. Software Eng., vol. 41, no. 11, pp. 1119-1137, 2015.

[67] A. Bertolino, G. De Angelis, B. Miranda, and P. Tonella, "Run java applications and test them in-vivo meantime," in 2020 IEEE 13th International Conference on Software Testing, Validation and Verification (ICST), pp. 454-459, IEEE, 2020.

[68] A. E. Genç, H. Sözer, M. F. Kıraç, and B. Aktemur, "Advisor: An adjustable framework for test oracle automation of visual output systems,'
IEEE Transactions on Reliability, 2019.

[69] S. Joshi and A. Orso, "Scarpe: A technique and tool for selective capture and replay of program executions," in 2007 IEEE International Conference on Software Maintenance, pp. 234-243, IEEE, 2007.

[70] J. Steven, P. Chandra, B. Fleck, and A. Podgurski, "jrapture: A capture/replay tool for observation-based testing," in Proc. of ISSTA, pp. 158-167, 2000.

[71] A. Saieva, S. Singh, and G. Kaiser, "Ad hoc test generation through binary rewriting," in 2020 IEEE 20th International Working Conference on Source Code Analysis and Manipulation (SCAM), pp. 115-126, IEEE, 2020.

[72] M. Gittens, K. Romanufa, D. Godwin, and J. Racicot, "All code coverage is not created equal: a case study in prioritized code coverage," in Proceedings of the 2006 conference of the Center for Advanced Studies on Collaborative research, pp. 11-es, 2006. 